\title{
Thermal Conductivity Equations via the Improved Adomian Decomposition Methods
}

\author{
Ashenafi Gizaw Jije \\ Department of Mathematics, Faculty of Natural and Computational Sciences, Gambella University, Gambella, Ethiopia
}

Email address:

ashugizaw98@gmail.com

\section{To cite this article:}

Ashenafi Gizaw Jije. Thermal Conductivity Equations via the Improved Adomian Decomposition Methods. Applied and Computational Mathematics. Vol. 9, No. 3, 2020, pp. 30-55. doi: 10.11648/j.acm.20200903.11

Received: April 1, 2020; Accepted: May 11, 2020; Published: May 27, 2020

\begin{abstract}
Several mathematical models that explain natural phenomena are mostly formulated in terms of nonlinear differential equations. Many problems in applied sciences such as nuclear physics, engineering, thermal management, gas dynamics, chemical reaction, studies of atomic structures and atomic calculations lead to singular boundary value problems and often only positive solutions are vital. However, most of the methods developed in mathematics are used in solving linear differential equations. For this reason, this research considered a model problem representing temperature distribution in heat dissipating fins with triangular profiles using MATLAB codes. MADM was used with a computer code in MATLAB to seek solution for the problem involving constant and a power law dependence of thermal conductivity on temperature governed by linear and nonlinear BVPs, respectively, for which considerable results were obtained. A problem formulated dealing with a triangular silicon fin and more examples were solved and analyzed using tables and figures for better elaborations where appreciable agreement between the approximate and exact solutions was observed. All the computations were performed using MATHEMATICA and MATLAB.
\end{abstract}

Keywords: Fins, Adomian Decomposition, Thermal Conductivity Equation, Nonnlinear

\section{Introduction}

\subsection{Background of the Study}

Several mathematical models that explain natural phenomena are mostly formulated in terms of nonlinear differential equations, both ordinary and partial. Many problems in applied sciences such as nuclear physics, engineering, thermal management, gas dynamics, chemical reaction, studies of atomic structures and atomic calculations lead to singular boundary value problems and often only positive solutions are important. However, most of the methods developed in mathematics are used in solving linear differential equations. For these reasons, a semi-analytical method named Adomian decomposition method (ADM) proposed by George Adomian $(1923-1990)$ has been attracting the attentions of many mathematicians, physicists, engineers and various graduate researchers. The method has the advantage of converging to the exact solution and can easily handle a wide class of both linear and nonlinear differential and integral equations. The commonly existing problems in various fields of studies mentioned earlier lead to singular BVPs of the form:

$$
u^{\prime \prime}(x)+t(x) u^{\prime}(x)+s(x) f(u(x))=r(x), x \in(a, b)
$$

subject to given boundary conditions where atleast one of the functions $t(x), s(x)$ and $r(x)$ has a singular point and $a, b, \alpha$ and $\beta$ are finite constants.

For example, when $t(x)=r(x)=0, s(x)=-x^{-1 / 2}$ and $f(u(x))=[u(x)]^{3 / 2},(1)$ is known as the Thomas-Fermi equation given by the singular equation $u^{\prime \prime}=x^{-1 / 2} u^{3 / 2}$ which arises in the study of electrical potential in an atom. Also when $t(x)=0$ and $f(u(x))=[u(x)]^{-\sigma},(1)$ is known as the generalized Emden-Fowler equation with a negative exponent and it frequently arises in applied mathematics. Another example is given by the singular equation $u^{\prime \prime}+$ $\frac{k}{x} u^{\prime}+g(u)=0$, sometimes written in the form $\left(x^{k} u^{\prime}\right)^{\prime}=$ $F(x, u)$ which results from an analysis of heat conduction through a solid with heat generation. The function $g(u)$ represents the heat generation in the solid, $u$ is the temperature and the constant $k$ is equal to 0,1 or 2 depending 
on whether the solid is a plate, a cylinder or a sphere. Equation (1) subject to boundary conditions represents temperature distribution inside a triangular extended surface (or fin) with thermal conductivity. Thus, extended surfaces are governed by BVPs; and they are widely used in many engineering appliances which include, but are not limited to, air conditioning, refrigeration, automobile and chemical processing equipments. For this reason this research tried to consider both invariant and power-law dependence of thermal conductivities governed respectively by linear and nonlinear BVPs.

The primary objective of using extended surfaces is to enhance the heat transfer rate between a solid and an adjoining fluid. Such an extended surface is termed a fin. In a convectional heat exchanger heat is transferred from one fluid to another through a metallic wall. The rate of heat transfer is directly proportional to the extent of the wall surface, the heat transfer coefficient and to the temperature difference between one fluid and the adjacent surface. If thin strips (fins) of metals are attached to the basic surface, extending into one fluid, the total surface for heat transfer is thereby increased.

This research tried to consider two triangular profile fin problems by incorporating with MATLAB codes. The modified Adomian decomposition method (MADM) is used to get the component decomposition terms. The Adomian decomposition method (ADM) is a well-known systematic method for practical solution of linear or nonlinear and deterministic or stochastic operator equations, including ODEs, PDEs, integro-differential equations, etc. The method is a powerful technique, which provides efficient algorithms for analytic approximate solutions and numeric simulations for real-world applications in the applied sciences and engineering. It permits to solve both nonlinear IVPs and BVPs without restrictive assumptions such as required by linearization, perturbation, discretization, guessing the initial term or a set of basis functions, and so forth. The accuracy of the analytic approximate solutions obtained can be verified by direct substitution. A key notion is the Adomian polynomials, which are tailored to the particular nonlinearity to solve nonlinear operator equations. The decomposition method has been used extensively to solve effectively a class of linear and nonlinear ordinary and partial differential equations. However, a little attention was devoted for its application in solving the singular two-point boundary value problems (STPBVPs). This project treated some classes of singular second-order two-point boundary value problems both analytically and numerically using the ADM and the modifications Improved Adomian Decomposition Method (IADM) and MADM focusing on the Dirchlet and mixed boundary conditions; and applied the symbolic softwares MATLAB and MATHEMATICA to facilitate computing.

\subsection{Statement of the Problem}

Numerous methods have been applied to determine numerical solutions of scientific problems that obviously result in variety of approximate solutions.
This research tried to investigate analytical and numerical solutions thermal conductivity governed generally by:

$$
u^{\prime \prime}(x)+t(x) u^{\prime}(x)+s(x) f(u(x))=r(x), x \in(a, b)
$$

where either of $t, s$ or $r$ are singular with given boundary conditions using the ADM, MADM and IADM accordingly. To perform this, it was tried to answer the research questions:

1. How effective are the decomposition method and its modifications to treat nonlinearity behaviors in thermal conductivity problems?

2. How do the modifications remove the singularity nature of the problems?

\subsection{Objectives}

The main objective of this research was to treat thermal conductivity equations through the improved ADMs. Specifically speaking, the study:

1) determined approximate solutions of thermal conductivity equations using improved ADMs.

2) performed error analysis to compare the results obtained accordingly.

3) made comparison between the numerical and analytical solutions of the problems by using plots of the equations on the same coordinate plane.

\section{Literature Review}

\subsection{Extended Surfaces}

Scientists and engineers are interested in singular BVPs because the problems arise in a wide range of applications, such as in chemical engineering, mechanical engineering, nuclear industry, thermal management and nonlinear dynamical systems. For this reason, these kinds of problems have been studied by many researchers. To be specific, as extended surfaces are found in many engineering appliances, numerous studies have been performed on different fin configurations with constant and variable thermal properties. Fins are echo-friendly and economic means of convective heat transfer enhancement. They are encountered quite often in practice: from industrial compact heat exchangers to $\mathrm{CPU}$ heat sink modules of personal computers. Finned structures, better known as heat sinks, have well served thermal management of electronic systems for many years. Kraus, A. D., Aziz, A. and Welty, J. provided a review on this subject devoted to various aspects of extended surface convective heat transfer [13]. Mokheimer Esmail, M. A. investigated the performance of annular fins with different profiles subject to variable heat transfer coefficient [17]. The performance of the fin is expressed in terms of fin efficiency as a function of the ambient and fin geometry parameters.

\subsection{Numerical and Analytical Treatments of the Bvps}

Zavalani, G. used Galerkin-finite element method (GFEM) to treat two point BVPs numerically [22]. The method uses three basic procedures. Making weak formulation is the 
first step to determine, the second step is to develop finite element formulation. Lastly, weighted average is used for full discretization; that are all required by neither the ADM nor its modifications. Moreover, the method fails to treat nonlinear and singular BVPs.

Khuri, S. A. and Sayfy, A. proposed a new approach, modified decomposition method in combination with the cubic B-spline collocation technique introduced for numerical solution of a class of singular boundary value problems arising in physiology [11]. The main thrust of this approach is to decompose the domain of the problem into two subintervals. The singularity, which lies in the first subinterval, is removed via the application of a modified decomposition procedure based on a special integral operator that is applied to surmount the singularity. Then, in the second sub-domain, which is outside the vicinity of the singularity, the resulting problem is treated employing the Bspline collocation technique. The approach is applicable to treat nonlinear singular BVPs and in addition, the approximate solutions converge faster to the exact solution but very vast computational work is needed.

Noor, M. A. and Mohyud-Din, S. T. used modified variational iteration method (MVIM) to determine solutions of singular and non-singular initial and boundary value problems [18]. The proposed technique is applied on equations of higher order boundary layer problem, unsteady flow of gas, third-order dispersive and fourth-order parabolic partial differential equations. The authors made the modification by introducing ADM to VIM. They used the Pade approximants technique (PAT) in order to make their work more concise and for better understanding of the solution behavior but needs caution to select the initial value because the approximants are heavily dependent on the initial value. Though they concluded that the proposed frame work is very powerful and efficient in finding the analytical solutions for singular and nonsingular boundary value problems, the method requires a huge computational work as it is incorporated from the ADM, VIM, and PAT.

Ravi, A. S. V. and Aruna, K. used an analytical method called DTM to obtain the exact solutions for some linear twopoint boundary value problems with singular nature [19]. Although these analytical methods are effective in the linear case, their applicability for nonlinear problems was not examined in the paper.

\subsection{Treatments of Related Problems Using ADM and Its Modifications}

Adomian formally introduced ADM concept and formulas for generating Adomian polynomials for all forms of nonlinearities since early 1980 's. Several authors have been focusing on this area to develop a practical method for the calculation of Adomian polynomials.

Adomian, G. reviewed the method in Applied Mathematics; and concluded that the decomposition method can be an effective method for solution of a wide class of problems providing generally a rapidly convergent series solution [1]. Based on the author, it has some distinct advantages over usual approximation methods in that it is computationally convenient, provides analytic, verifiable solutions not requiring perturbation, linearization, or discretization required by methods like FDM which results in massive computations.

Inc, M. and Evans, D. J. obtained an approximate solution for only one nonlinear example by using the ADM-Padê technique [9]. The technique they proposed requires the computation of undetermined coefficients.

Hasan, Y. Q. and Zhu, L. M.. introduced an efficient modification of the ADM for solving singular initial value problem in the second-order ordinary differential equations [8]. The scheme is tested for some examples and the obtained results demonstrate efficiency of the proposed method. The study showed that the decomposition method is simple and easy to use and produces reliable results with few iterations. The results show that the rate of convergence of the modified decomposition method, MADM is higher than the standard $\mathrm{ADM}$ for initial value problems.

Several other researchers also have developed modifications to the ADM. The modifications arise from evaluating difficulties specific for the type of problem under consideration. The modification usually involves only a slight change and is aimed at improving the convergence or accuracy of the series solution.

Duan, J.-S, Rach, R., Baleanu, D. and Wazwaz, A.-M. reviewed the $\mathrm{ADM}$ and its modifications including different modified and parameterized recursion schemes, the multistage ADM for initial value problems as well as the multistage ADM for boundary value problems, new developments of the method and its applications to linear or nonlinear and ordinary or partial differential equations, including fractional differential equations [5]. The authors presented a contemporary review of the ADM and discussed its utility and advantages for solving linear or nonlinear and deterministic or stochastic operator equations without any restrictive assumptions, including ODEs, PDEs, integral equations and integro-differential equations for IVPs or BVPs. They also concluded that the ADM is the method of choice for solving nonlinear differential equations with a wide class of analytic nonlinearities including product, polynomial, exponential, trigonometric, hyperbolic, composite, negative-power, radical and decimal-power nonlinearities. Furthermore, the ADM has been shown to be a reliable method for the solution of nonlinear fractional ODEs and PDEs for both IVPs and BVPs. In summary, based on Duan, J.-S, Rach, R., Baleanu, D. and Wazwaz, A.-M. the $\mathrm{ADM}$ is a powerful and efficient technique for the solution of nonlinear ordinary, partial and fractional differential equations [5]. It provides the analyst with an easily computable, readily verifiable and rapidly convergent sequence of analytic approximate functions for the solution.

Ravi, A. S. V. and Aruna, K. proposed two modified recursive schemes for solving a class of doubly singular twopoint boundary value problems [20]. The authors illustrated how the modified recursive schemes can be used to solve a class of doubly singular two-point boundary value problems. 
Based on the authors the accuracy of the numerical results indicates that the method is well suited for the solution of this type problem. They included that the main advantage of the approach is that it provides a direct scheme to obtain approximate solutions, and they have also shown graphically that these approximate solutions are almost identical to the analytic solution. They also stated another advantage of the modified recursion scheme as: it does not require the computation of undetermined coefficients, whereas most of previous recursive schemes like by Inc, M. and Evans, D. J. require the computation of undetermined coefficients [9]. The method provides a reliable technique which requires less work compared to the traditional techniques such as FDM, Cubic spline method, and standard ADM. The numerical results of the examples are presented and they believe that only a few terms are required to obtain accurate solutions. By comparing the results with other existing methods, it has been proved that proposed modified ADM is a more powerful method for solving the singular problems.

Lin, Y. and Chen, C. K. proposed a modification of the Adomian decomposition method, MADM [16]. The authors demonstrated that nonlinear double singular BVPs of second order can be handled without difficulty. Furthermore, the authors stated that MADM can also be used to solve singular boundary value problems where the traditional method ADM fails. The numerical computation gives a more precise approximation of the solution. The reported results show a greater improvement over the traditional method.

Kaliyappan, M. and Hariharan, S. recall that they presented a simple way of computing Adomian polynomials by applying the decomposition of positive integers as a subscript of the variable $u$ for nonlinear terms [10]. They developed MATLAB codes that can generate Adomian polynomials with single variable. This paper used MATHEMATICA instead.

\section{Materials and Methods}

1) convectional heat transfer is considered to study temperature distribution along a triangular fin profile by formulating a singular two-point BVP.

2) relevant journals and books were addressed to gather information about singular BVPs and the methods to treat the problems.

3) nonlinear expressions were decomposed by the help of MATHEMATICA using Adomian decomposition formula:

$$
A_{n}=\frac{1}{n !} \frac{d^{n}}{d \lambda^{n}}\left[N\left(\sum_{i=0}^{n} \lambda^{i} u_{i}\right)\right]_{\lambda=0}, n=0,1,2, \ldots
$$

4) The inverse operator,

$$
L^{-1}(.)=\int_{b}^{x} x^{n-k-1} \int_{0}^{x} x^{k-n} \int_{0}^{x} \ldots \int_{0}^{x} x(.) d x \ldots d x
$$

in its reduced form was incorporated in to MATLAB codes to treat constant and power-law dependent thermal conductivity problems. Operators like

$$
\begin{gathered}
L^{-1}{ }_{x x}(.)=\int_{x_{0}}^{x} \int_{x_{0}}^{x}(.) d x d x-\frac{x-x_{0}}{1-x_{0}} \int_{x_{0}}^{1} \int_{x_{0}}^{x}(.) d x d x, \\
L_{t}^{-1}=\int_{0}^{t}(.) d t
\end{gathered}
$$

were used in IADM to treat problems with Dirchlet boundary conditons. (described in subsection 5.1)

5) Furtheremore, MATLAB and MATHEMATICA were applied suitably to facilitate the computations with the improved operators; and graphs were plotted using those programs.

\section{Preliminaries}

\subsection{Singular Points}

It has been required to solve the differential equation

$$
P(x) u^{\prime \prime}(x)+Q(x) u^{\prime}(x)+R(x) u(x)=0
$$

or, in standard form,

$$
u^{\prime \prime}(x)+t(x) u^{\prime}(x)+s(x) u(x)=0
$$

in the neighborhood of a singular point, as the behavior of the solutions there may be among their most important features. When the singularities are not too wild, a modification of the technique of power series can be used to calculate the solutions there.

To simplify the discussion, attentions are restricted to equations of the form (6) where, $P, Q$ and $R$ are polynomials, that they may be assumed to have no common factors.

The solutions to the linear second order differential equations of the form (6) in the case with $P, Q$, and $R$ real analytic in a neighborhood of a point $x=x_{0}$ and $P \neq 0$ can be expanded as power series of the form:

$$
u(x)=\sum_{n} c_{n} x^{n}, n=0,1,2,3, \ldots
$$

and with the knowledge of $c_{0}$ and $c_{1}$, the coefficients $c_{i}$ with $i \geq 2$ can be determined successively by certain recurrence relations.

There are many important linear second order differential equations (with non-constant coefficients) that arise in mathematical physics which do not satisfy the above conditions, and power series solutions would like to be able to obtained for some of these.

The method discovered by the German mathematician F. G. Frobenius in the 1870's and is therefore often called the Frobenius method is used to determine the singular points.

Definition 4.1. A function $f$ is said to be analytic at a point $x_{0} \in I$ if $f$ can be expanded in a power series about $x_{0}$ which has a positive radius of convergence.

Definition 4.2. The point $x=x_{0}$ is called a singular point of (4.1) if $P\left(x_{0}\right)=0$.

This causes difficulties in writing (6) in the standard form $u^{\prime \prime}+\frac{Q(x)}{P(x)} u^{\prime}+\frac{R(x)}{P(x)} u=0$.

Definition 4.3. The singular point $x=x_{0}$ is said to be 
regular singular point of (6) if the equation can be written as

$$
u^{\prime \prime}+\frac{Q(x)}{P(x)} u^{\prime}+\frac{R(x)}{P(x)} u=0
$$

where, $\lim _{x \rightarrow x_{0}} \frac{\left(x-x_{0}\right) Q(x)}{P(x)}$ and $\lim _{x \rightarrow x_{0}} \frac{\left(x-x_{0}\right)^{2} R(x)}{P(x)} \quad$ both exist and are finite.

This means that, for $x$ near $x_{0}$, the functions $\frac{Q(x)}{P(x)}$ and $\frac{R(x)}{P(x)}$ can be rewritten as $\frac{Q(x)}{P(x)}=\frac{\left(Q_{1}(x)\right.}{\left(x-x_{0}\right)}$ and $\frac{R(x)}{P(x)}=\frac{R_{1}(x)}{\left(x-x_{0}\right)^{2}}$ where $Q_{1}(x)$ and $R_{1}(x)$ are analytic at $x=x_{0}$. This is the same as saying that the multiplier of $u^{\prime}$ in (9) has at most the factor $\left(x-x_{0}\right)$ in denominator; and the multiplier of $u$ in (9) has at most the factor $\left(x-x_{0}\right)^{2}$ in the denominator.

A singular point which is not regular is called irregular singular point.

Remark: If $P(x)$ and $Q(x)$ are analytic at $x_{0}$ and $P\left(x_{0}\right) \neq$ 0 , then $\frac{Q(x)}{P(x)}$ is analytic at $x_{0}$.

Example 4.1. Given the Legendre differential equation $\left(1-x^{2}\right) u^{\prime \prime}-2 x u^{\prime}+\alpha(\alpha+1) u=0$, let $\alpha \in \mathbb{R}, \alpha \neq 0$. Find the singular points of the equation and determine which regular singular points are.

Solution

The points are the zeros of $\left(1-x^{2}\right)=(1-x)(1+x)$ and are clearly, $x=-1, x=1$.

To test whether example (4.1) is regular, divide the given equation by the factor $\left(1-x^{2}\right)$ to obtain

$$
u^{\prime \prime}-\frac{2 x u^{\prime}}{(1-x)(1+x)}+\frac{\alpha(\alpha+1)}{(1-x)(1+x)} u=0
$$

Here, it can be observed that the multiplier of $u^{\prime}$ has the form

$$
\frac{1}{x-1}\left(\frac{2 x}{1+x}\right)
$$

and the multiplier of $u$ has the form

$$
\frac{1}{x-1}\left(\frac{-\alpha(\alpha+1)}{1+x}\right) \text {. }
$$

The denominators of the factors in the parentheses above are not zero at $x=1$, so they are analytic at $x=1$. Hence, $x=1$ is a regular singular point. A similar test for $x=-1$ shows that it also is a regular singular point.

\subsection{Boundary Value Problems}

Definition 4.4. A boundary value problem for second order ordinary differential equations is a differential equation with conditions specified at the extremes of the independent variable in the equation.

Definition 4.5 . Let $f: \mathbb{R}^{3} \rightarrow \mathbb{R}$ be given function and $u_{0}$ and $u_{1}$ be given numbers. The problem

$$
\begin{gathered}
u^{\prime \prime}=f\left(x, u, u^{\prime}\right), x \in(a, b) \\
u(a)=u_{0}, u(b)=u_{1}
\end{gathered}
$$

is called two-point boundary value problem. Two-point boundary value problems are BVPs with boundary conditions given at two specified points.

Linear BVPs can mainly be classified as homogeneous and inhomogeneous. They are called homogeneous if $g(x), u_{0}$ and $u_{1}$ are all zero. They are said to known as inhomogeneous if atleast one of $g(x), u_{0}$ and $u_{1}$ are nonzero.

A boundary value problem for a differential equation consists of finding a solution $u$ of the differential equation that also satisfies the boundary conditions. A boundary value problem may or may not have a solution; and if it has a solution it may be unique or infinitely many.

Definition 4.6. A two-point BVP with a singular point $x=x_{0}$ subjected to two specified boundary conditions at $a$ and $b$ in an interval $\mathrm{I}$ as $u(a)=\alpha$ and $u(b)=\beta$, where $a, b, \alpha$ and $\beta$ are some constants, the two-point BVP is called a STPBVP.

\subsection{Operators}

An operator is a function that takes a function as an argument instead of numbers as it is used to dealing with in functions. Here are some examples of operators.

$$
L=\frac{d}{d x} \quad L=\frac{\partial}{\partial x} \quad L=\int d x \quad L=\int_{a}^{b} d x
$$

Or, if a function is plugged in, say $u(x)$, in each of the above, then the following can be obtained.

$$
L(u)=\frac{d u}{d x} L(u)=\frac{\partial u}{\partial x} L(u)=\int u(x) d x L(u)=\int_{a}^{b} u(x) d x
$$

These are all fairly simple examples of operators but the derivative and integral are operators. A more complicated operator would be the heat operator. The heat operator can be found from a slight rewrite of the heat equation without sources. The heat operator is then

$$
L=\frac{\partial}{\partial t}-k \frac{\partial^{2}}{\partial x^{2}}
$$

and

$$
L=\frac{d}{d x}\left(\frac{1}{x} \frac{d}{d x}\right)
$$

is another differential operator for a particular second order differential equation (see the example below).

The operator $L$ in second order differential equations is a twice differentiable function. The domain of $L$ is the twice differentiable functions on an open interval $I$. The terminology $L$ of the function $u$ is used to describe $L(u)$ or $L[u]$ or, simply $L u$ and the range of the functions on $I$ (and hence $L[u]$ is itself a function on $I$ ). Generally, $L$ is chosen

$$
L[.]=\frac{d^{p}}{d x^{p}}[.]
$$

for the $p^{\text {th }}$ order differential equations and thus its inverse $L^{-1}$ follows as the $p$-fold definite integration operator from $x_{0}$ to $x$. The operator $L$ defined has the following basic property: 
If $u_{1}$ and $u_{2}$ are twice differentiable functions on $I$ and $c_{1}$ and $c_{2}$ are constants, then

$$
L\left[c_{1} u_{1}+c_{2} u_{2}\right]=c_{1} L\left[u_{1}\right]+c_{2} L\left[u_{2}\right]
$$

Definition 4.7. An operator $L$ satisfying property (17) is called linear operator.

Example 4.2. The differential equation

$$
u^{\prime \prime}-\frac{1}{x} u^{\prime}=0
$$

can be re written in compact form as

$$
\left(\frac{1}{x} u^{\prime}\right)^{\prime}=0
$$

So from this an operator can be generated to be:

$$
L=\frac{d}{d x}\left(\frac{1}{x} \frac{d}{d x}\right)
$$

so that (18) can be written in an operator form as:

$$
L[u]=\frac{d}{d x}\left(\frac{1}{x} \frac{d u}{d x}\right)
$$

Beyond this, it can be verified that the operator $L$ is a linear operator. Bearing the basic properties of derivation of ordinary differential equations in mind and plugging in the expression $c_{1} u_{1}+c_{2} u_{2}$ into the operator above it can be shown as:

$$
\begin{gathered}
L\left[c_{1} u_{1}+c_{2} u_{2}\right]=\frac{d}{d x}\left(\frac{1}{x} \frac{d}{d x}\left(c_{1} u_{1}+c_{2} u_{2}\right)\right) \\
=\frac{d}{d x}\left[\frac{1}{x} \frac{d}{d x}\left(c_{1} u_{1}\right)+\frac{1}{x} \frac{d}{d x}\left(c_{2} u_{2}\right)\right] \\
=\frac{d}{d x}\left(c_{1} \frac{1}{x} \frac{d}{d x} u_{1}+c_{2} \frac{1}{x} \frac{d}{d x} u_{2}\right) \\
=c_{1} \frac{d}{d x}\left(\frac{1}{x} \frac{d u_{1}}{d x}\right)+c_{2} \frac{d}{d x}\left(\frac{1}{x} \frac{d u_{2}}{d x}\right) \\
=c_{1} L\left[u_{1}\right]+c_{2} L\left[u_{2}\right]
\end{gathered}
$$

Thus the operator at (19) is linear operator.

An operator that is not linear is known as nonlinear operator. In this research nonlinear operators in which nonlinear functions are plugged are symbolized by some various representations like $N u$ and $p(u)$. These operators are used to determine the Adomian polynomials by the help of Adomian formula which is briefly discussed in the following chapter.

\section{Thermal conductivity equations via Adm, Iadm and Madm}

\subsection{General Description of the Adomian Decomposition Method}

In the 1980's, George Adomian introduced a new method to solve nonlinear functional equations. This method has since been termed the ADM and has been the subject of many investigations. The method involves splitting the equation under investigation into linear and nonlinear portions. The linear operator representing the linear portion of the equation is inverted and the inverse operator is then applied to the equation. The nonlinear portion is decomposed into a series of Adomian polynomials. This method generates a solution in the form of a series whose terms are determined by a recursive relationship using the polynomials. In reviewing the basic methodology involved, consider a general differential equation in an operator form:

$$
L u+R u+N u=r
$$

where $L$ is an operator representing the linear portion which is easily invertible, $N$ is the nonlinear operator representing the nonlinear term and $R$ is a linear operator for the remainder of the linear portion. Applying the inverse operator $L^{-1}$, the equation then becomes

$$
L^{-1} L u=L^{-1} r-L^{-1} R u-L^{-1} N u
$$

Since $L$ is linear, $L^{-1}$ would represent integration and with any given boundary conditions, will give an equation for $u$ incorporating these conditions. This gives

$$
u(x)=g(x)-L^{-1} R u-L^{-1} N u
$$

where $g(x)$ represents the function generated by integrating $r$ and using the boundary conditions.

The Adomian decomposition method introduces the solution $u(x)$ and the nonlinear function $N u$ by the infinite series as:

$$
u(x)=\sum_{i=0}^{\infty} u_{n}(x)
$$

and

$$
N u=\sum_{i=0}^{\infty} A_{n}\left(u_{0}, u_{1}, u_{2}, \ldots, u_{n}\right)
$$

where, $A_{n}$ are the Adomian polynomials that can be determined by Adomian formula:

$$
A_{n}=\frac{1}{n !} \frac{d^{n}}{d \lambda^{n}}\left[N\left(\sum_{i=0}^{n} \lambda^{i} u_{i}\right)\right]_{\lambda=0}, n=0,1,2, \ldots
$$

So for $n=0,(26)$ reduces to

$$
A_{0}=N\left(u_{0}\right)
$$

For $n=1$, it reduces to

$$
\begin{gathered}
A_{1}=\frac{d}{d \lambda}\left[N\left(u_{0}+\lambda u_{1}\right)\right]_{\lambda=0} \\
\Rightarrow A_{1}=u_{1} N^{\prime}\left(u_{0}\right)
\end{gathered}
$$

For $n=2$, it becomes

$$
\begin{gathered}
A_{2}=\frac{1}{2 !} \frac{d^{2}}{d \lambda^{2}}\left[N\left(u_{0}+\lambda u_{1}+\lambda^{2} u_{2}\right)\right]_{\lambda=0} \\
\Rightarrow A_{2}=u_{2} N^{\prime}\left(u_{0}\right)+\frac{1}{2 !} u_{1}^{2} N^{\prime \prime}\left(u_{0}\right)
\end{gathered}
$$


The desired Adomian polynomials can be determined using similar procedure.

$$
A_{3}=u_{3} N^{\prime}\left(u_{0}\right)+u_{1} u_{2} N^{\prime \prime}\left(u_{0}\right)+\frac{1}{3 !} u_{1}^{3} N^{\prime \prime \prime}\left(u_{0}\right)
$$

It can be observed that $A_{0}$ depends only on $u_{0}, A_{1}$ depends only on $u_{0}$ and $u_{1}, A_{2}$ depends only on $u_{0}, u_{1}$ and $u_{2}$ and so on.

Optionally, a simple way of computing Adomian polynomials of any type of nonlinearity is presented by applying the decomposition of positive integers $n$ as a subscript of the variable $u$ for nonlinear terms through the use of MATHEMATICA software in the appendix C. MATHEMATICA exploits general symbolic programming for generating Adomian polynomials.

Now, substituting (24) and (25) into (23), one can get:

$$
\sum_{i=0}^{\infty} u_{n}(x)=g(x)-L^{-1} \sum_{i=0}^{\infty} R u_{n}-L^{-1} \sum_{i=0}^{\infty} A_{n}
$$

The recursive relationship is found to be

$$
\begin{gathered}
u_{0}=g(x) \\
u_{n+1}=-L^{-1} R u_{n}-L^{-1} A_{n}
\end{gathered}
$$

And hence from (28),

$$
\begin{gathered}
u_{0}=g(x) \\
u_{1}=-L^{-1} R u_{0}-L^{-1} A_{0} \\
u_{2}=-L^{-1} R u_{1}-L^{-1} A_{1} \\
\cdot \\
\cdot \\
u_{i}=-L^{-1} R u_{i-1}-L^{-1} A_{i-1}
\end{gathered}
$$

for $i=1,2,3, \ldots$. So, having determined the components $u_{n}, n \geq 0$ the solution $u$ in a series form follows immediately by (24).

For instance, as a simple example consider the nonlinear differential equation:

$$
u^{\prime}+u^{2}=1, u(0)=0
$$

with exact solution $u(x)=\frac{1-e^{-2 x}}{1+e^{2 x}}$.

Recall that the ADM involves separating the equation under investigation into linear and nonlinear portions. The linear operator representing the linear portion of the equation is inverted and the inverse operator is then applied to the equation. Any given conditions are taken into consideration. The nonlinear portion is decomposed into a series of Adomian polynomials. This method generates a solution in the form of a series whose terms are determined by a recursive relationship using these Adomian polynomials.

Following the method described above, the desired linear operator is defined as

$$
L=\frac{d}{d x}
$$

The inverse operator is then

$$
L^{-1}=\int_{0}^{x}(.) d x
$$

Rewriting (29) in operator form, we have

$$
\begin{gathered}
L u+N u=1 \\
\Rightarrow L u=1-N u
\end{gathered}
$$

where $N$ is nonlinear operator such that $N u=u^{2}$.

Next apply the inverse operator for $L$ to the left hand side of the equation the following way

$$
L^{-1} L u=u(x)-u(0)
$$

Using the initial condition this becomes

$$
L^{-1} L u=u(x)
$$

Returning this to (33) it becomes

$$
\begin{gathered}
u(x)=L^{-1}(1)-L^{-1}(N u) \\
=\int_{0}^{x} d x-L^{-1}(N u)=x-L^{-1}(N u)
\end{gathered}
$$

Next the Adomian polynomials, $A_{n}$ would be generated as follows:

Let $u$ be expanded as infinite series

$$
u(x)=
$$

and define

$$
N u=\sum_{n=0}^{\infty} A_{n}
$$

Then

$$
\sum_{n=0}^{\infty} u_{n}(x)=x-L^{-1}\left(\sum_{n=0}^{\infty} A_{n}\right)
$$

So from (29) the ADM invites the recursive relation:

$$
\begin{gathered}
u_{0}(x)=x \\
u_{n+1}(x)=-L^{-1}\left(A_{n}\right), n \geq 0
\end{gathered}
$$

Now using the Adomian formula given by (26) the polynomials become:

$$
\begin{gathered}
A_{0}=\left.N\left(u_{0}\right)\right|_{\lambda=0}=u_{0}^{2} \\
A_{1}=\frac{d}{d \lambda}\left(N\left(u_{0}+\lambda u_{1}\right)\right)=2 u_{0} u_{1} \\
A_{2}=\frac{d}{d \lambda}\left(N\left(u_{0}+\lambda u_{1}+\lambda^{2} u_{2}\right)\right)=2 u_{0} u_{2}+u_{1}^{2}
\end{gathered}
$$


Using these results in the recursive relation (26) the solution is generated as follows: $u_{0}(x)=x$

$$
\begin{gathered}
u_{1}(x)=-L^{-1}\left(A_{0}\right) \\
=-L^{-1}\left(u_{0}^{2}\right) \\
=-\int_{0}^{x} x^{2} d x=-\frac{1}{3} x^{3} \\
u_{2}(x)=-L^{-1}\left(A_{1}\right) \\
=-L^{-1}\left(2 u_{0} u_{1}\right) \\
=-\int_{0}^{x} 2 x\left(-\frac{1}{3} x^{3}\right) d x=\frac{2}{15} x^{5}
\end{gathered}
$$

Hence the approximate solution for $u(x)$ obtained using the first five components denoted $\phi_{5}$ look like the following.

$$
\begin{gathered}
\phi_{5}=u_{0}+u_{1}+u_{2}+u_{3}+u_{4} \\
=x-\frac{1}{3} x^{3}+\frac{2}{15} x^{5}-\frac{17}{315} x^{7}+\frac{62}{2835} x^{9} .
\end{gathered}
$$

The decomposition method and its modifications can be applied suitably to treat singular BVPs. How the ADM treats a class of singular BVPs is discussed below. For illustrative purpose, the following class of singular BVPs is considered which can be handled more easily, quickly and elegantly by implementing $\mathrm{ADM}$ for the exact solutions without making massive computational work. To begin,

Consider (1) in the form

$$
\left(x^{\alpha} u^{\prime}\right)^{\prime}=F(x, u), x \in(a, b)
$$

subject to the boundary conditions

$$
u(a)=\omega \text { and } u(b)=\beta
$$

Equation (41) in an operator form can be rewritten as:

$$
L(u)=F(x, u)
$$

where the linear differential operator $L$ is defined by

$$
L(.)=\frac{d}{d x}\left(x^{\alpha} \frac{d}{d x}[.]\right)
$$

The inverse operator $L^{-1}$ is therefore defined by

$$
L^{-1}(.)=\int_{0}^{x}\left(x^{-\alpha} \int_{0}^{x}[.] d x\right) d x
$$

Operating $L^{-1}$ with (43), it then follows

$$
u=u(0)+\int_{0}^{x}\left(x^{-\alpha} \int_{0}^{x}[F(x, u)] d x\right) d x
$$

In this equation the expression $F(x, u)$ may represent linear or nonlinear differential equation. Note that a second order ordinary differential equation written in standard form over the dependent variable $u$ and independent variable $x$ is said to be linear if it can be written in the form:

$$
u^{\prime \prime}+t(x) u^{\prime}+s(x) u=r(x)
$$

otherwise it can be called as nonlinear.

It is not unusual to transform an equation into another equivalent form so that it would be convenient to use better techniques of solving. The following two cases deal with the expression $F(x, u)$ in (46).

Case 1. If $F(x, u)$ is linear.

Consider $F(x, u)$ in the form:

$F(x, u)=h(x)+r(x) u$. Thus, (46) becomes

$u=u(0)+\int_{0}^{x}\left(x^{-\alpha} \int_{0}^{x}[h(x)] d x\right) d x+\int_{0}^{x}\left(x^{-\alpha} \int_{0}^{x}[r(x) u] d x\right) d x$

Now decompose the solution $u$ as in (24),

It is important to note that the standard ADM suggests that the zeroth component $u_{0}$ usually defined by the function:

$$
g(x)=u(0)+\int_{0}^{x}\left(x^{-\alpha} \int_{0}^{x}[h(x)] d x\right) d x
$$

that represents the terms arising from integrating the source term $h(x)$ and from using the given conditions; and all are assumed to be prescribed. Accordingly, the solution can be computed by using the recurrence relation:

$$
\begin{gathered}
u_{0}=g(x) \\
u_{n+1}=\int_{0}^{x}\left(x^{-\alpha} \int_{0}^{x}\left[r(x) u_{n}\right] d x\right) d x, n \geq 0
\end{gathered}
$$

Case 2. If $F(x, u)$ is nonlinear.

Consider $F(x, u)$ in the form:

$$
F(x, u)=r(x) p(u)
$$

where $p(u)$ is nonlinear.

Substituting this in to (46),

$$
u=u(0)+\int_{0}^{x}\left(x^{-\alpha} \int_{0}^{x}[r(x) p(u)] d x\right) d x
$$

The ADM is based on decomposing $u$ and the nonlinear term $p(u)$ as

$$
u(x)=\sum_{n=0}^{\infty} u_{n}(x)
$$

and

$$
p(u)=\sum_{i=0}^{\infty} A_{n}\left(u_{0}, u_{1}, u_{2}, \ldots, u_{n}\right)
$$

Using these results in (51), it then follows

$$
\sum_{n=0}^{\infty} u_{n}=u(0)+\sum_{n=0}^{\infty} \int_{0}^{x}\left(x^{-\alpha} \int_{0}^{x}\left[r(x) A_{n}\right] d x\right) d x, n \geq 0
$$

According to the standard ADM, the solution can be computed by using the recurrence relation:

$$
u_{0}=u(0)
$$




$$
u_{n+1}=\int_{0}^{x}\left(x^{-\alpha} \int_{0}^{x}\left[r(x) A_{n}\right] d x\right) d x, n \geq 0
$$

Thus, determining the components $u_{n}, n \geq 0$ using (49) and/or (55) the solution $u$ in a series form follows immediately. The series may be summed to provide the solution in a closed form. However, for concrete problems, the $n$ term partial sum may be used to give the approximate solution as:

$$
\Phi_{n}=\sum_{i=0}^{n-1} u_{i}
$$

The classical ADM is very powerful in treating nonlinear BVPs, though this is one of the qualities of the method over some other methods it has its own shortcomings like its failure to treat some nonlinear singular boundary value problems. The following subsection addresses some merits and demerits of the method.

\subsection{1. $M A D M$}

Since the introduction of the method in early 1980's, ADM has led to several modifications made by various researchers in an attempt to improve the accuracy and expand the application of the original method. As pointed out above, the rate of convergence of the series solutions is one of the potential shortcomings of the decomposition method. To improve on this, the authors tried to introduce different modifications of the method. To begin with, based on Ravi, A. S. V. and Aruna, K. the standard ADM is modified in such a way that the function $g$ in (16) can be divided into two parts as follows to increase rate of convergence of the series solution and minimize the size of computations [21]. This modification is applicable irrespective of the types of the BVPs under consideration.

$$
g=g_{0}+g_{1}
$$

Accordingly, a slight variation was proposed only on the components $u_{0}$ and $u_{1}$. The suggestion was that only the parts $g_{0}$ be assigned to the component $u_{0}$, whereas the remaining part $g_{1}$ be combined with other terms given in (21) to define $u_{1}$ to get the recursive relation:

$$
\begin{aligned}
& u_{0}=g_{0} \\
& u_{1}=g_{1}-L^{-1} R u_{0}-L^{-1} A_{0} \\
& u_{n+1}=-L^{-1}\left(R u_{n}\right)-L^{-1}\left(A_{n}\right), n \geq 1
\end{aligned}
$$

Although this variation in the formation of $u_{0}$ and $u_{1}$ is slight, however it plays a major role in accelerating the convergence of the solution and in minimizing the size of calculations. In many cases the modified scheme avoids unnecessary computations, especially in calculation of the Adomian polynomials. In other words, sometimes there is no need to evaluate the so-called Adomian polynomials required for nonlinear operators or if needed to evaluate these polynomials the computation will be reduced very considerably by using the modified recursive scheme. There are two important remarks related to the modified method. First, by proper selection of the functions $g_{0}$ and $g_{1}$, the exact solution $u$ may be obtained by using very few iterations, and sometimes by evaluating only two components. The success of this modification depends only on the choice of $g_{0}$ and $g_{1}$, and this can be made through trials, that are the only criteria which can be applied so far. Second, if $g$ consists of one term only, the scheme (28) should be employed in this case.

Another modification of the standard ADM which alleviates the deficiency of treating some singular boundary value problems like, BVPs subjected to the form of mixed boundary conditions is MADM presented by Hasan, Y. Q. and $\mathrm{Zhu}, \mathrm{L}$. M. [8]. In fact, it is a slight refinement to the original ADM; it only modifies the involved differential operator. Generally, MADM by the authors mentioned proposes the differential and inverse operators:

$$
L=x^{-1} \frac{d^{n-1}}{d x^{n-1}} x^{n-k} \frac{d}{d x} x^{k-n+1} \frac{d}{d x}(.)
$$

and

$$
\begin{gathered}
L^{-1}(.)=\int_{b}^{x} x^{n-k-1} \int_{0}^{x} x^{k-n} \int_{0}^{x} \ldots \int_{0}^{x} x(.) d x \ldots d x \\
n-1 \text { times }
\end{gathered}
$$

for treatment of $n+1$ order boundary value problem of the form:

$$
u^{(n+1)}+\frac{k}{x} u^{(n)}+N u=f n=0,1,2, \ldots ; k=0,1, \text { or } 2
$$

The operators above are reduced so as to treat second order BVPs and incorporate symbolic programmings into them to obtain solutions to a triangular fin problems involving both constant and power-law dependent thermal conductivity (highly nonlinear).

Kim, W. and Chun, C. came up with another modification of the standard ADM to solve singular $n+1$ order boundary value problems [12]. This scheme is designed in such a way that BVPs with singular nature can easily be treated. In this project the operators are used to treat singular second-order BVPs with mixed boundary conditions. Generally, to see what MADM by the authors mentioned look like, consider the singular boundary value problem of $n+1$ order ordinary differential equation (61) given the following way:

$$
\begin{gathered}
u^{(n+1)}+\frac{k}{x} u^{(n)}+N u=f(x) \\
u(0)=a_{0}, u^{\prime}(0)=a_{1}, \ldots, u^{r-1}(0)=a_{r-1}, \\
u(b)=c_{0}, u^{\prime}(b)=c_{1}, \ldots, u^{n-r}(b)=c_{n-r}
\end{gathered}
$$

Where $N$ is nonlinear differential operator of order less than $n, f(x)$ is a given function, $a_{0}, a_{1}, \ldots, a_{r-1}, c_{0}, c_{1}, \ldots, c_{n-r}, b$ are given constants, where $k \leq r \leq n, r \geq 1$.

Now (62) can be re written in the form

$$
x^{-2} \frac{d^{n-1}}{d x^{n-1}}\left[x^{2} u^{\prime \prime}+(k-2 n+2) x u^{\prime}\right]+N u=f
$$

Or equivalently,

$$
x^{-2} \frac{d^{n-1}}{d x^{n-1}}\left[x^{2 n-k} \frac{d}{d x}\left(x^{k-2 n+2} \frac{d u}{d x}\right)\right]+N u=f
$$

(64) can be written in the operator form 


$$
L_{2} L_{1} u=f(x)-N u
$$

Where, the differential operator $L$ employs the first two derivatives

$$
\begin{gathered}
L_{1}=x^{2 n-k} \frac{d}{d x}\left(x^{k-2 n+2} \frac{d}{d x}\right) \\
L_{2}=x^{-2} \frac{d^{n-1}}{d x^{n-1}}
\end{gathered}
$$

in order to overcome the singularity behavior at $x=0$.

In view of (67) and (68) the inverse operators $L_{1}^{-1}$ and $L_{2}^{-1}$ are the integral operators defined by

$$
\begin{gathered}
L_{1}^{-1}=\int_{0}^{x} x^{2 n-k-2} \int_{b}^{x} x^{k-2 n}(.) d x d x \\
L_{2}^{-1}=\int_{0}^{x} \ldots \int_{0}^{x} x^{2}(.) d x \ldots d x \\
n-1 \text { time }
\end{gathered}
$$

By applying $L_{2}^{-1}$ on (63), one can have

$$
L_{1} u=\Psi_{1}(x)+L_{2}^{-1} f(x)-L_{2}^{-1} N u
$$

such that

$$
L_{2} \Psi_{1}(x)=0
$$

By applying $L_{1}^{-1}$ on (71), one can have

$$
u(x)=\Psi_{2}(x)+L_{1}^{-1} \Psi_{1}(x)+L_{1}^{-1} L_{2}^{-1} f(x)-L_{1}^{-1} L_{2}^{-1} N u
$$

such that

$$
L_{1} \Psi_{2}(x)=0
$$

The standard ADM introduces the solution $u(x)$ and the nonlinear function $\mathrm{Nu}$ by infinite series given by (24) and (25) where the Adomian polynomials are determined by the formula at (27). Substituting (24) and (25) in to (73) gives

$$
\begin{gathered}
\sum_{n=0}^{\infty} u_{n}=\Psi_{2}(x)+L_{1}^{-1} \Psi_{1}(x)+L_{1}^{-1} L_{2}^{-1} f(x)- \\
L_{1}^{-1} L_{2}^{-1} \sum_{n=0}^{\infty} A_{n}
\end{gathered}
$$

Identifying $u_{0}=\Psi_{2}(x)+L_{1}^{-1} \Psi_{1}(x)+L_{1}^{-1} L_{2}^{-1} f(x)$, the Adomian method admits the use of the recursive relation

$$
\begin{gathered}
u_{0}=\Psi_{2}(x)+L_{1}^{-1} \Psi_{1}(x)+L_{1}^{-1} L_{2}^{-1} f(x) \\
u_{n+1}=-L^{-1} A_{n}
\end{gathered}
$$

which gives

$$
\begin{aligned}
& u_{0}=\Psi(x)+L^{-1} f(x) \\
& u_{1}=-L^{-1} A_{0} \\
& u_{2}=-L^{-1} A_{1} \\
& u_{3}=-L^{-1} A_{2}
\end{aligned}
$$

This leads to the complete determination of the components $u_{n}$ of $u(x)$. The series solution $u(x)$ defined by (24) follows immediately. For numerical purposes, the $n$ term approximant defined by (56) can be used to approximate the exact solution.

\subsubsection{IADM}

Ebaid, A. 2010. made improvements of operators developed earlier by Lesnic, D. 2001 for the purpose of treating the heat equation with Dirchlet boundary condition [6], [15]. In this work the IADM is used to deal with linear and nonlinear STPBVPs with Dirchlet boundary conditions. The improvement is based on the ADM and Lesnic's work later developed by Ebaid, A. 2010 [6]. Lesnic, D. 2001. proposed the inverse operators [15]:

$$
\begin{gathered}
L^{-1}{ }_{x x}(.)=\int_{x_{0}}^{x} \int_{x_{0}}^{x}(.) d x d x-\frac{x-x_{0}}{1-x_{0}} \int_{x_{0}}^{1} \int_{x_{0}}^{x}(.) d x d x, \\
L_{t}^{-1}=\int_{0}^{t}(.) d t
\end{gathered}
$$

to solve the Dirchlet BVP for the heat equation

$$
u_{t}=u_{x x}, x_{0}<x<1, t>0
$$

under the boundary conditions $u\left(x_{0}, t\right)=f_{0}(t), u(1, t)=$ $f_{1}(t)$ and the initial condition $u(x, 0)=p(x)$.

Using the definition in (78) it is observed that

$$
L^{-1}{ }_{x x}\left(u_{x x}\right)=u(x, t)-u\left(x_{0}, t\right)-\frac{x-x_{0}}{1-x_{0}}\left[u(1, t)-u\left(x_{0}, t\right)\right]
$$

i.e., the boundary conditions can be used directly. However, from (78) again one can see that the lower bound of all integrations is restricted to the initial point $x_{0}$.

In fact, this restriction can be avoided by using a new definition of $L^{-1} x x$ which gives the same result as in (80) and given by:

$$
L^{-1}{ }_{x x}(.)=\int_{x_{0}}^{x} \int_{c}^{x}(.) d x d x-\frac{x-x_{0}}{1-x_{0}} \int_{x_{0}}^{1} \int_{c}^{x}(.) d x d x
$$

where, $c$ is free lower point. This free lower point plays an important role if the equation being solved has a singular point. So the desired operator originally designed by the author mentioned earlier is derived as follows.

$L^{-1}{ }_{x x}($.$) is defined as:$

$$
L^{-1}{ }_{x x}(.)=\int_{a}^{x} \int_{c}^{x}(.) d x d x-z(x) \int_{d}^{b} \int_{e}^{x}(.) d x d x
$$

where $z(x)$ is to be determined such that $L^{-1}{ }_{x x}\left(u^{\prime \prime}(x)\right)$ can be expressed only in terms of the boundary conditions given in (2). Using this definition, thus:

$$
\begin{gathered}
L_{x x}^{-1}\left(u^{\prime \prime}(x)\right)=u(x)-u(a)-(x-a) u^{\prime}(c)- \\
z(x)\left[u(b)-u(d)-(b-d) u^{\prime}(e)\right] . \\
=u(x)-u(a)-z(x)[u(b)-u(d)]-(x-a) u^{\prime}(c)+ \\
z(x)[(b-d)] u^{\prime}(e) .
\end{gathered}
$$

Setting $d=a$ and $e=c$,

$$
L^{-1} x x\left(u^{\prime \prime}(x)\right)=u(x)-u(a)-z(x)[u(b)-u(a)]-
$$




$$
(x-a) u^{\prime}(c)+z(x)(b-a) u^{\prime}(c) .
$$

In order to express $L^{-1}{ }_{x x}\left(u^{\prime \prime}(x)\right)$ in terms of the two boundary conditions only, the coefficient $u^{\prime}(c)$ has to be eliminated by setting

$$
\begin{gathered}
-(x-a) u^{\prime}(c)+z(x)\left[(b-a) u^{\prime}(c)\right]=0 \text { assuming } \\
u^{\prime}(c) \neq 0 . \\
\Rightarrow z(x)=\frac{x-a}{b-a}
\end{gathered}
$$

Using (84) in (82), the operator below proposed by Ebaid, A. 2010 is obtained to solve the singular two-point Dirchlet BVPs [6].

$$
L_{x x}^{-1}(.)=\int_{a}^{x} \int_{c}^{x}(.) d x d x-\frac{x-a}{b-a} \int_{a}^{b} \int_{c}^{x}(.) d x d x, a \neq b, c-
$$

Thus (83) is reduced to:

$$
L^{-1}{ }_{x x}\left(u^{\prime \prime}(x)\right)=u(x)-u(a)-\frac{x-a}{b-a}[u(b)-u(a)]
$$

From ( 85$)$ it is noted that $L_{x x}^{-1}\left(u^{\prime \prime}(x)\right)$ is already expressed in terms of the given boundary conditions without any restrictions on $c$. So, the choice of the value that $c$ can take depend properly on the singular point of the equation under consideration. For example, if the equation has a singular point say at $x=x_{0}, c$ will be chosen to be any real value except the value of $x_{0}$. Moreover, if the equation has two singular points at $x=x_{1}$ and $x=x_{2}$, then $c$ will be considered any real value except these values of $x_{1}$ and $x_{2}$. In general, if the equation has $n$ singular points $x_{1}, x_{2}, \ldots, x_{n}$, then $c$ takes any real value except the values of these singular points.

For solving linear and non-linear singular two-point boundary value problems under the Dirchlet boundary condition, IADM is established using (85) together with the standard ADM.

Consider (1) in the form:

$$
u^{\prime \prime}(x)=r(x)-t(x) u^{\prime}(x)-s(x) f(u(x))
$$

Applying the operator $L^{-1}{ }_{x x}($.$) given by (86) on both$ sides of (72), it is observed that:

$$
\begin{aligned}
u(x)= & u(a)+\frac{x-a}{b-a}[u(b)-u(a)]+L^{-1}{ }_{x x}[r(x)] \\
& -L^{-1}{ }_{x x}\left[t(x) u^{\prime}(x)\right]-L^{-1}{ }_{x x}[s(x) f(u(x))] .
\end{aligned}
$$

The Adomian decomposition method introduces the solution $u(x)$ and the nonlinear function $f(u)$ by infinite series as in (24) and (25), respectively.

Substituting the results into (88) and according to the ADM, the solution $u(x)$ can be smartly computed by using the recurrence relations constructed based on the following cases.

Case 1:- If $f(u)=u$, i.e., linear function, then the solution $u(x)$ can be computed by using the recurrence relation:

$$
\begin{gathered}
u_{0}(x)=u(a)+\frac{x-a}{b-a}[u(b)-u(a)]+L^{-1}{ }_{x x}[r(x)] \\
u_{n+1}(x)=-L_{x x}^{-1}\left[t(x) u_{n}^{\prime}(x)+s(x) u_{n}(x)\right], n \geq 0
\end{gathered}
$$

Case 2:- If $f(u)$ is nonlinear function, then the recurrence relation required to compute the solution $u(x)$ is:

$$
\begin{gathered}
u_{0}(x)=u(a)+\frac{x-a}{b-a}[u(b)-u(a)]+L^{-1}{ }_{x x}[r(x)] \\
u_{n+1}(x)=-L_{x x}^{-1}\left[t(x) u_{n}^{\prime}(x)+s(x) A_{n}(x)\right], n \geq 0
\end{gathered}
$$

Where the pairs of equations (89) and (85), (90) and (85)improve the standard ADM, (IADM) and can be used to solve linear and nonlinear singular two-point boundary value problems subject to Dirchlet boundary conditions. Hence, using the recurrence relation (85) or (86) depending on linearity behavior of the boundary value problem, the $n$-term truncated approximate solution can be computed by the help of (56).

\subsection{Numerical and Analytical Illustrations}

Triangular fin problem that involves both invariant and power-law dependent thermal conductivities is considered in this section. The modified operators by Hasan, Y. Q. and Zhu, L. M. shown in sub section 5.1.3 and MATLAB codes are assimilated to obtain the numerical solutions [8]. For the sake of illustrations, additional examples are shown.

\subsubsection{A Fin Problem}

Consider a wall at temperature $T_{b}$ transferring heat by convection to an ambient at temperature $T_{\infty}(\operatorname{Tinf})$ as shown in the figure below.

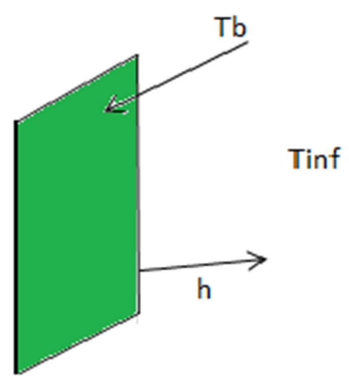

Figure 1. A wall transferring heat by convection.

The rate of heat transfer from this wall may be evaluated in terms of a heat transfer coefficient in the form

$$
q=h A\left(T_{b}-T_{\infty}\right)
$$

where, $q$ is the heat flux, $h$ coefficient of heat transfer, $A$ the cross section, $T_{b}$ temperature at the base /wall temperature/ and $T_{\infty}$ the ambient temperature.

One of the prime objectives of the study of heat transfer is to find ways of controlling this $q$. For example, the design of a heat exchanger is often based on achieving the smallest possible heat transfer area or the largest possible amount of heat transfer for any given size heat exchanger. 
As can be observed from (91) one of the ways of increasing $q$ is by increasing the heat transfer area. The surface area of a wall may, in principle, be increased in two ways as shown in figures below.

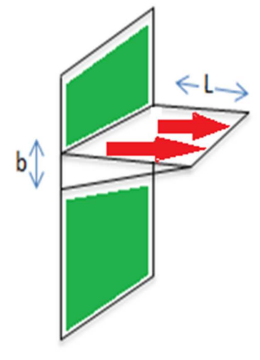

a) Triangular profile

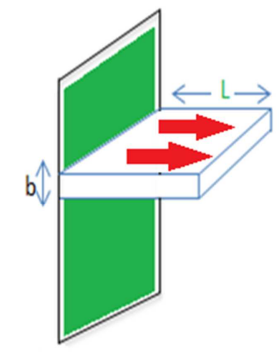

b) Rectangular profile

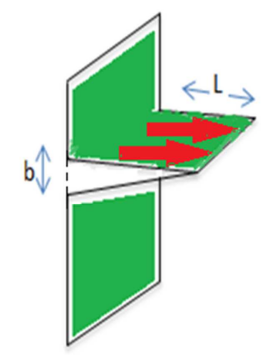

c) Triangular profile
Figure 2. Triangular and rectangular fin profiles.

In Figure 2(c) the extended surfaces are integral parts of the base material, obtained by a casting or extruding process. In Figures 2( $a$ and $b)$ the extended surfaces, that may or may not be made from the base material, are attached to the base by pressing, soldering, or welding. The same geometry is obtained, though less frequently, by machining the base material. In practice, manufacturing technology and cost dictate the selection of the most desirable form.

Applications of extended surfaces are numerous, particularly in heat transfer to gaseous media. Since in this case the corresponding heat transfer coefficient is low, a small, compact heat exchanger may be achieved only by the use of extended surfaces.

Since the temperature of an extended surface does not remain constant along its length, because of transversal heat transfer by convection to the surroundings, the heat transfer from extended surfaces cannot be evaluated from (91). Thus it is unquestionable to evaluate the temperature distribution in extended surfaces, which enables to determine the heat transfer in terms of the temperature distribution. So this research studied the temperature distribution in a triangular profile as shown in figure below considering both constant and variable thermal conductivities across a variable cross section as follows.

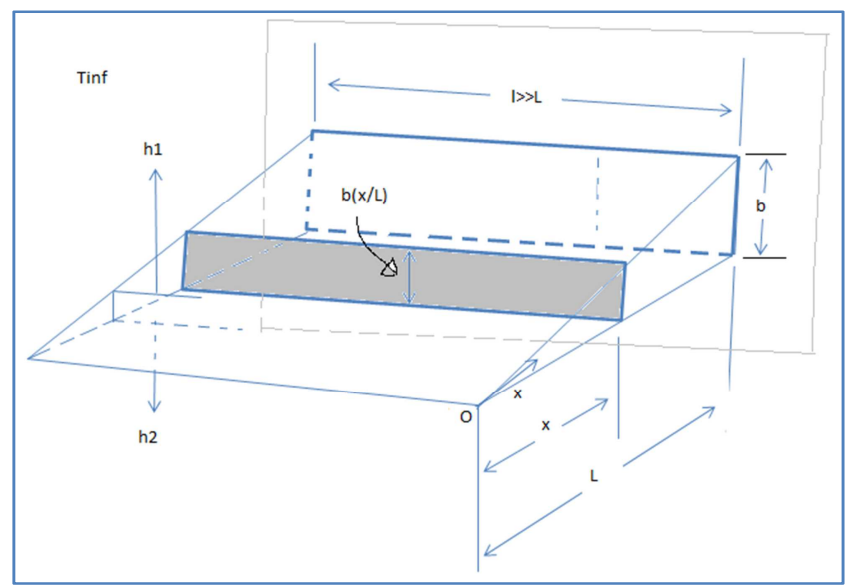

Figure 3. The geometry of a straight fin of triangular profile.
The general formulation of problems of extended surfaces with variable cross sections is given by

$$
\frac{d}{d x}\left(k A \frac{d T}{d x}\right)-h p\left(T-T_{\infty}\right)=0
$$

This equation is elaborated in two cases considering constant and variable thermal conductivities in the following sub sections.

\section{(i) Constant Thermal Conductivity}

Assuming constant thermal conductivity and measuring temperature above the ambient, (92) may further be redesigned to give:

$$
\frac{d}{d x}\left(k A \frac{d \theta}{d x}\right)-h p \theta=0
$$

where, $\theta=T-T_{\infty}$; and noting from figure above that $A=b\left(\frac{x}{L}\right) l$ and $h p=\left(h_{1}+h_{2}\right) l$, inserting these values into (5.64) it becomes

$$
\begin{gathered}
\frac{k b l}{L} \frac{d}{d x}\left(x \frac{d \theta}{d x}\right)-h p \theta=0 \\
\Rightarrow \frac{d}{d x}\left(x \frac{d \theta}{d x}\right)-\frac{\left(h_{1}+h_{2}\right) L}{k b} \theta=0
\end{gathered}
$$

Hence the differential equation governing temperature distribution inside a triangular fin with invariant thermal conductivity can be expressed as:

$$
\frac{d}{d x}\left(x \frac{d \theta}{d x}\right)-m^{2} \theta=0
$$

with boundary conditions $\left.\frac{d \theta}{d x}\right|_{x=0}=0$, and $\theta(L)=\theta_{b}$, where $\theta$ is the temperature measured above the ambient temperature, subscript $b$ stands for fin base and $L$ is the fin length. In addition, $m^{2}$ is the fin parameter defined as:

$$
m^{2}=\frac{\left(h_{1}+h_{2}\right) L}{k b}
$$

where $h_{1}$ and $h_{2}$ are convective heat transfer coefficients of fin's either sides, $k$ is the thermal conductivity and $b$ is fin's vertical dimension at its base. For this formulation, the origin of coordinates is placed at the tapered end of the fin.

From (59) and (60), appropriate differential and inverse operators to treat (95) respectively are,

$$
L_{x x}(.)=x^{-1} \frac{d}{d x} x \frac{d}{d x}(.)
$$

and

$$
L_{x x}^{-1}(.)=\int_{L}^{x} x^{-1} \int_{0}^{x} x(.) d x d x
$$

Equation (95) can be rewritten as:

$$
\frac{d^{2} \theta}{d x^{2}}+\frac{1}{x} \frac{d \theta}{d x}-m^{2} \frac{\theta}{x}=0
$$

This in its operator form can be expressed as:

$$
L_{x x} \theta=m^{2} \frac{\theta}{x}
$$


Applying (98) on both sides of (100), the following is found

$$
\theta(x)=\theta(L)+L_{x x}^{-1}\left(m^{2} \frac{\theta}{x}\right)
$$

Using (98) and the boundary condition, the recursive relation can easily be formulated as:

$$
\begin{gathered}
\theta_{0}=\theta_{b} \\
\theta_{k+1}=\int_{L}^{x} x^{-1} \int_{0}^{x} m^{2} \theta_{k} d x d x, k \geq 0
\end{gathered}
$$

MATLAB codes are used to determine the required decomposition components of this recurrence relation and are available in appendix A. For the sake of demonstration, the first six components of the solution computed by the foresaid MATLAB code are given as follows.

$$
\begin{gathered}
\theta_{0}=\theta_{b} \\
\theta_{1}=m^{2} \theta_{b} x-m^{2} \theta_{b} L \\
\theta_{2}=\frac{m^{4} \theta_{b}}{4} x^{2}-m^{4} \theta_{b} L x+\frac{3 m^{4} \theta_{b} L^{2}}{4} \\
=\theta_{b}\left(\left(m^{12}\left(\frac{90921 L^{6}-131436 L^{5} x+47475 L^{4} x^{2}-7600 L^{3} x^{3}+675 L^{2} x^{4}-36 L x^{5}+x^{6}}{518400}\right)\right)+\left(m^{8}\left(\frac{211 L^{4}-304 L^{3} x+108 L^{2} x^{2}-16 L x^{3}+x^{4}}{576}\right)\right)-\cdots+\right. \\
\left.\left(m^{16}\left(\frac{136407699 L^{8}-197216832 L^{7} x+71282064 L^{6} x^{2}-11449536 L^{5} x^{3}}{1625702400}+\frac{1033900 L^{4} x^{4}-59584 L^{3} x^{5}+2352 L^{2} x^{6}-64 L x^{7}+x^{8}}{1625702400}\right)\right)+1\right) .
\end{gathered}
$$

$$
\begin{gathered}
\theta_{3}=\frac{m^{6} \theta_{b}}{36} x^{3}-\frac{m^{6} \theta_{b} L}{4} x^{2}+\frac{3 m^{6} \theta_{b} L^{2}}{4} x-\frac{19 m^{6} \theta_{b} L^{3}}{36} \\
\theta_{4}=\frac{m^{8} \theta_{b}}{576} x^{4}-\frac{m^{8} \theta_{b} L}{36} x^{3}+\frac{3 m^{8} \theta_{b} L^{2}}{16} x^{2}-\frac{19 m^{8} \theta_{b} L^{3}}{36} x \\
+\frac{211 m^{8} \theta_{b} L^{4}}{576} \\
\theta_{5}=\frac{m^{10} \theta_{b}}{14400} x^{5}-\frac{m^{10} \theta_{b} L}{576} x^{4}+\frac{m^{10} \theta_{b} L^{2}}{48} x^{3} \\
-\frac{19 m^{10} \theta_{b} L^{3}}{144} x^{2}+ \\
\frac{211 m^{10} \theta_{b} L^{4}}{576} x-\frac{1217 m^{10} \theta_{b} L^{5}}{4800}
\end{gathered}
$$

So by (56) the approximate solution, say using the first ten components can be determined as:

But only the first three components of the MADM solution are used to compare with the exact solution. The relevant analytical exact solution of (95) as given by Arpaci, S. Vedat is [2]:

$$
\frac{\theta(x)}{\theta_{b}}=\frac{I_{0}\left(2 m x^{0.5}\right)}{I_{0}\left(2 m L^{0.5}\right)}
$$

where $I_{0}$ denotes Bessel's function of second kind. So from (88),

$$
\theta(x)=\theta_{b} \frac{I_{0}\left(2 m x^{0.5}\right)}{I_{0}\left(2 m L^{0.5}\right)}
$$

A relative error which covers part of a silicon fin length is defined to see how the approximate MADM solutions to the exact solution are.

An absolute relative deviation (ARD) from exact solution that considers ten different points of the interval is defined and computed the following way.

$$
\begin{gathered}
A R D=\frac{1}{10}\left(\left|\frac{\theta_{e}-\theta_{M A D M}}{\theta_{e}}\right|_{\text {at } x=L}+\left|\frac{\theta_{e}-\theta_{M A D M}}{\theta_{e}}\right|_{\text {at } x=0.95 L}+\left|\frac{\theta_{e}-\theta_{M A D M}}{\theta_{e}}\right|_{\text {at } x=0.9 L}+\left|\frac{\theta_{e}-\theta_{M A D M}}{\theta_{e}}\right|_{\text {at } x=0.85 L}+\left|\frac{\theta_{e}-\theta_{M A D M}}{\theta_{e}}\right|_{\text {at } x=0.8 L}+\right. \\
\left.\left|\frac{\theta_{e}-\theta_{M A D M}}{\theta_{e}}\right|_{\text {at } x=0.75 L}+\left|\frac{\theta_{e}-\theta_{M A D M}}{\theta_{e}}\right|_{\text {at } x=0.7 L}+\left|\frac{\theta_{e}-\theta_{M A D M}}{\theta_{e}}\right|_{\text {at } x=0.65 L}+\left|\frac{\theta_{e}-\theta_{M A D M}}{\theta_{e}}\right|_{\text {at } x=0.6 L}+\left|\frac{\theta_{e}-\theta_{M A D M}}{\theta_{e}}\right|_{a t x=0.55 L}\right) \\
=\frac{1}{10}(0.003000590579085+0.006144019569687+0.00944568578639900+0.0100292368369663891+ \\
0.01659947989466741+0.02049881777482788+0.02465295003258466+0.02910035359995550+ \\
0.03388917438611092)=0.0153360308460284
\end{gathered}
$$

ARD from exact solution is defined to see how far the MADM solutions from the exact solutions are; and it shows that the approximate solution digresses only about $1.5 \%$ from the exact solution for a small parameter $m$. Such a definition for ARD gives a global and average sense of deviation from exact solution and is more valid than focusing on error at a single fixed point. Even this gap may be filled more by taking appropriate values of the constants.

Note that larger temperature difference does not imply less heat loss. Arpaci, S. Vedat shares this by stating 'In terms of heat transfer from extended surfaces, it is more appropriate to compare the exact and the approximate heat losses than the 
exact and approximate temperatures, to determine the limitation of the approximate solution' [2].

\section{(ii) Power Law Dependence of Thermal Conductivity}

Assuming general power-law dependence under steady condition for thermal conductivity of the fin material in the form

$$
k=k_{0} T^{\beta}
$$

and averaged heat transfer coefficient, the succeeding governing equation can be derived the following way.

$$
\frac{d}{d x}\left(k A \frac{d T}{d x}\right)-2 h p\left(T-T_{\infty}\right)=0
$$

where, $A, T, p, h$ denote the variable cross-sectional area, local temperature, the periphery and the heat transfer coefficient, respectively. Substituting the appropriate terms, (107) becomes

$$
\begin{gathered}
\frac{d}{d x}\left(b\left(\frac{x}{L}\right) l\left(k_{0} T^{\beta}\right) \frac{d T}{d x}\right)-2 h l\left(T-T_{\infty}\right)=0 \\
\Rightarrow \frac{d}{d x}\left(x T^{\beta} \frac{d T}{d x}\right)-\frac{2 h L}{k_{0} b}\left(T-T_{\infty}\right)=0 \\
\Rightarrow x T^{\beta} \frac{d^{2} T}{d x^{2}}+T^{\beta} \frac{d T}{d x}+\beta x T^{\beta-1}\left(\frac{d T}{d x}\right)^{2}-\frac{2 h L}{k_{0} b}\left(T-T_{\infty}\right)=0
\end{gathered}
$$

Dividing by $x T^{\beta}$ and rearranging gives:

$$
\sum_{n=0}^{\infty} T_{n}=T(L)-\beta L_{x x}^{-1}\left(\sum_{n=0}^{\infty} A_{n}\right)+\frac{2 h L}{k_{0} b} L_{x x}^{-1}\left(\frac{1}{x} \sum_{n=0}^{\infty} B_{n}\right)-\frac{2 h L T_{\infty}}{k_{0} b} L_{x x}^{-1}\left(\frac{1}{x} \sum_{n=0}^{\infty} C_{n}\right)
$$

Thus, the recurrence relation needed is:

$$
\begin{aligned}
& T_{0}(x)=T(L) \\
& \quad T_{n+1}(x)=-\beta L_{x x}^{-1}\left(A_{n}\right)+\frac{2 h L}{k_{0} b} L_{x x}^{-1}\left(\frac{1}{x} B_{n}\right)-\frac{2 h L T_{\infty}}{k_{0} b} L_{x x}^{-1}\left(\frac{1}{x} C_{n}\right) ; n \geq 0
\end{aligned}
$$

To reach the very final solution, each decomposed component of the series

$$
T=\sum_{n=0}^{\infty} T_{n}
$$

has to be computed recursively. For this reason, it is necessary to obtain Adomian polynomials components of $A_{n}, B_{n}$, and $C_{n}$ at each of the iterations. To handle this task neatly, three functions returning symbolic representations for $A_{n}, B_{n}$, and $C_{n}$, a function to take inverse transform and a core code to calculate the ultimate solution with the help of these functions are used. All these MATLAB codes are given in the appendix B.

Using the computational code mentioned previously the MADM solution series can be expanded upto any desired component. To demonstrate, the first three components of the decomposition solution can be shown as:

$$
\begin{gathered}
T_{0}=T_{b} \\
T_{1}=\frac{2 h L}{k_{0} b} T_{b}^{1-\beta}(x-L)-\frac{2 h L T_{\infty}}{k_{0} b} T_{b}^{-\beta}(x-L)
\end{gathered}
$$

$$
\begin{gathered}
T_{2}=\frac{h^{2} L^{2} T_{b}^{1-2 \beta}}{k_{0}^{2} b^{2}}\left(-T_{b}^{2} x^{2}+4 T_{b}^{2} x L+T_{b} x^{2} T_{\infty}-4 T_{b}^{2} x L \beta-2 T_{b} x^{2} \beta T_{\infty}+8 T_{b} x L \beta T_{\infty}-3 T_{b}^{2} L^{2}+3 T_{b} T_{\infty} L^{2}+3 T_{b}^{2} L^{2} \beta-\right. \\
\left.6 \beta T_{b} T_{\infty} L^{2}+\beta T_{\infty}^{2} x^{2}-\beta T_{\infty}^{2} x L+3 \beta T_{\infty}^{2} L^{2}\right) .
\end{gathered}
$$

\subsubsection{Numerical and Analytical Treatments}

\section{Model problem}

Silicon, being an efficient thermal conductor, has been of extensive interest in fabrication of cooling fins and packed heat sink modules especially for thermal management in microelectronics. For temperatures ranging within $300-$
$1400 \mathrm{~K}$, a power law correlation for thermal conductivity of silicon is given by:

$$
k=k_{300}\left(\frac{T}{300}\right)^{\alpha}
$$

where, $k_{300}=148 \frac{\mathrm{W}}{\mathrm{Km}}, \alpha=-1.3$ 
Regarding (106), $k_{0}=148 \times 300^{1.3} \frac{W K^{0.3}}{m}$

Now it can be taken the advantage of the described computational work in section 5.2 based on MADM to investigate the temperature distribution in a triangular silicon fin with dimensions $L=0.05 \mathrm{~m}$ and $b=0.005 \mathrm{~m}$, subject to a constant base temperature of $T_{b}=423 K\left(150 C^{0}\right)$, and ambient temperature of $T_{\infty}=298 K\left(25 C^{0}\right)$. The results to this model problem from the first eight decomposition solutions by MADM are used to plot the graph as shown in figure 4 .

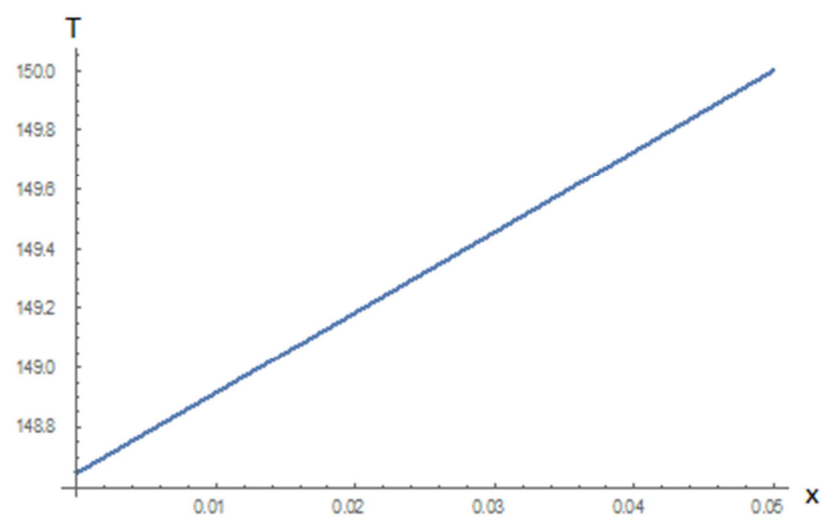

Figure 4. Temperature distribution along the length of the silicon fin.

The figure is all about variant thermal conductivity (the power law dependence) of the silicon fin considered above taking the first eight decomposition solutions of the problem. It can be observed from the figure that the temperature distribution decreases as one goes from the source to the tapered end of the triangular fin. So, unnecessary heat from a system can be dissipated to the surrounding atmosphere through extended surfaces. Compared to the FDM by Mokheimer Esmail, M. A temperature along the fin determined by MADM is observed to decline faster though only the first eight components of the decomposition were used [17]. Hence, overheating of operating machines can be protected by plugging in many extended surfaces to the machines. All these confirm that fins can serve as effective coolants. Outside this, it is not difficult to understand how powerful the scheme, modification of ADM to treat highly nonlinear BVPs.

Example 5.1. Consider

$$
x^{2} u^{\prime \prime}(x)-x u^{\prime}(x)+u(x)=0
$$

$1 \leq x \leq 2$ with the conditions $u(1)=1$ and $u(2)=1$.

\section{ADM solution}

As the given equation is not inhomogeneous, $\mathrm{ADM}$ fails to provide the self-cancelling noise terms that ADM favors where sum of noise terms vanishes in the limit. Self- cancelling noise terms appear for some inhomogeneous differential equations only. Thus, if terms in $u_{0}$ are cancelled by terms in $u_{1}$, even though $u_{1}$ includes further terms, then the remaining non cancelled terms in $u_{1}$ are cancelled by terms in $u_{2}$, the remaining terms of $u_{2}$ are cancelled by some terms of $u_{3}$ and so on. Finally, the exact solution of the equation is readily found for the inhomogeneous case by determining the first two or three terms of the solution $u(x)$ and by keeping only the non-cancelled terms of $u_{0}$.

Moreover, it can be observed that the standard ADM fails to treat such problems since its operators are designed so as to give no attention to the boundary conditions of the BVPs. Such difficulties are resolved perfectly by the IADM in which the operators are designed in such a way that all the boundary conditions are included. But the exact solution of the problem as given by Arpaci, S. Vedat is [2]

$$
u(x)=\frac{2 x \ln 2-x \ln x}{2 \ln 2}
$$

\section{IADM solution}

Rewriting (119) in the standard form gives:

$$
u^{\prime \prime}(x)-\frac{1}{x} u^{\prime}(x)+\frac{1}{x^{2}} u(x)=0
$$

With

$$
\begin{gathered}
p(x)=-\frac{1}{x}, q(x)=\frac{1}{x^{2}}, r(x)=0, a=1, b=2, u(1) \\
=1, u(2)=1
\end{gathered}
$$

using (89) given by

$$
\begin{gathered}
u_{0}(x)=u(a)+\frac{x-a}{b-a}[u(b)-u(a)]+L_{x x}^{-1}[r(x)] \\
u_{n+1}(x)=-L_{x x}^{-1}\left[t(x) u_{n}^{\prime}(x)+s(x) u_{n}(x)\right], n \geq 0
\end{gathered}
$$

Substituting the given terms, one can get:

$$
u_{0}=1+L_{x x}^{-1}[0]=1
$$

For $n=0$ and the given terms above at the second equation in (120), reduces to:

$$
\begin{gathered}
u_{1}=-L_{x x}^{-1}\left[-\frac{1}{x} u_{0}^{\prime}(x)+\frac{1}{x^{2}} u_{0}(x)\right] \\
=-L_{x x}^{-1}\left[\frac{1}{x^{2}}\right] \\
\Rightarrow u_{1}=-(-1+x) \ln 2+\ln x
\end{gathered}
$$

Similarly, $u_{2}$ can be determined as:

$$
\begin{gathered}
u_{2}=L_{x x}^{-1}\left[\frac{1}{x} u_{1}^{\prime}(x)-\frac{1}{x^{2}} u_{1}\right] \\
\Rightarrow u_{2}=-L_{x x}^{-1}\left[\frac{1}{x} \frac{d}{d x}(-(\ln 2)(x-1)+\ln x)-\frac{1}{x^{2}}(-(\ln 2)(x-1)+\ln x)\right]
\end{gathered}
$$




$$
u_{2}=-\frac{1}{2}(\ln 2)^{2}+\frac{1}{2}(-1+x)(\ln 2)^{2}+\frac{1}{2}(1-x)(\ln 4)^{2}+\frac{1}{2}(\ln 2 x)^{2}
$$

By (56),

$$
\Rightarrow \Phi_{3}=1+(1-x) \ln 2+\ln x+\frac{1}{2}(-2+x)(\ln 2)^{2}+\frac{1}{2}(1-x)(\ln 4)^{2}+\frac{1}{2}(\ln 2 x)^{2}
$$

which is the approximate solution using the first three iterations only. But the first seven iterative results are used to get better numerical results in the table 1 that shows the numerical illustration using both the IADM and exact solutions together.

In order to verify how close the IADM solution $\Phi_{3}$ to the exact solution $u(x)$, the graph of the two solutions are plotted in figure 5. For the IADM solution it is enough to use only the first three components of the decomposition series solution. All the computations and the plot are done by MATHEMATICA.

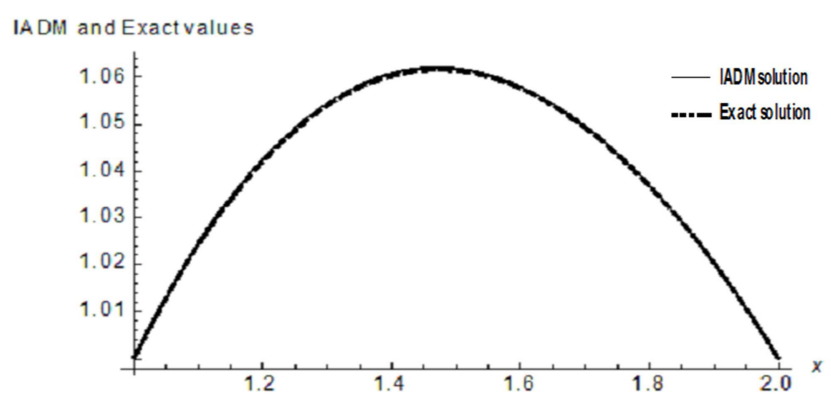

Figure 5. Comparison of the IADM and Exact solutions for example 5.1.

Table 1. Numerical results for example 5.1.

\begin{tabular}{llll}
\hline $\mathbf{x}$ & $\begin{array}{l}\text { Approximate } \\
\text { Solution }\end{array}$ & Exact Solution & Error $/ \boldsymbol{u}(\boldsymbol{x})-\boldsymbol{\Phi}_{\boldsymbol{I A D M} /}$ \\
\hline 1 & 1 & 1 & 0 \\
1.1 & 1.024373035 & 1.024373062 & $2.6258 \mathrm{E}-08$ \\
1.2 & 1.042179302 & 1.042179356 & $5.3793 \mathrm{E}-08$ \\
1.3 & 1.053967377 & 1.053967445 & $6.7857 \mathrm{E}-08$ \\
1.4 & 1.060201157 & 1.060201221 & $6.3608 \mathrm{E}-08$ \\
1.5 & 1.061278079 & 1.061278124 & $4.4892 \mathrm{E}-08$ \\
1.6 & 1.057542456 & 1.057542476 & $2.0090 \mathrm{E}-08$ \\
1.7 & 1.049295464 & 1.049295466 & $1.8739 \mathrm{E}-09$ \\
1.8 & 1.03680277 & 1.036802784 & $1.4216 \mathrm{E}-08$ \\
1.9 & 1.030300538 & 1.020300552 & $1.3555 \mathrm{E}-08$ \\
2 & 1 & 1 & 0 \\
\hline
\end{tabular}

In figure 5, the IADM solution $\Phi_{3}$ and the exact solution

$$
u(x)=\frac{(2 \ln 2) x-x \ln x}{2 \ln 2}
$$

$$
\begin{aligned}
u_{1}=-x-( & \left.-\frac{11}{12}+\frac{11 \mathrm{e}}{12}\right) x+\frac{\mathrm{e} x}{2}+\frac{x^{2}}{4}+\frac{\mathrm{e} x^{2}}{4}-\frac{x^{3}}{6}+\frac{\mathrm{e} x^{3}}{6}+x \ln x-\frac{1}{2} \mathrm{e} x \ln x \\
u_{2}=-\frac{x}{24}- & \left(-\frac{233}{360}-\frac{169 \mathrm{e}}{720}\right) x-\frac{5 \mathrm{e} x}{24}-\frac{25 x^{2}}{48}-\frac{\mathrm{e} x^{2}}{24}-\frac{13 x^{3}}{144}-\frac{\mathrm{e} x^{3}}{48}+\frac{x^{4}}{72}+\frac{\mathrm{e} x^{4}}{36}-\frac{x^{5}}{120}+\frac{\mathrm{e} x^{5}}{120}+\frac{1}{24} x \ln x+\frac{5}{24} \mathrm{e} x \ln x \\
& +\frac{1}{4} x^{2} \ln x-\frac{1}{8} \mathrm{e} x^{2} \ln x+\frac{1}{6} x^{3} \ln x-\frac{1}{12} \mathrm{e} x^{3} \ln x-\frac{1}{4} x(\ln x)^{2}+\frac{1}{8} \mathrm{e} x(\ln x)^{2}
\end{aligned}
$$

are plotted together but to get better approximate solution, MATHEMATICA is used to evaluate up to the $7^{\text {th }}$ iteration as shown in table 1. It can be concluded from the figure that an accurate IADM solution is obtained considering only few terms of the decomposition solution. Also, in order to compare the approach with another modification of the ADM at Benabidallah, M. and Cherruault, $\mathrm{Y}$ the numerical results for the absolute errors $/ u(x)-\Phi_{I A D M}(x) /$ are presented in table 1 [3]. In the journal mentioned, the authors used the 7terms of the ADM in indirect way in which a huge amount of computational work is needed to obtain the numerical solution with absolute errors $\leq 6 \times 10^{-4}$. Hence, in comparison, the improved Adomian decomposition method is not only more accurate but also more simple and direct. Example 5.1. Consider the linear singular equation

$u^{\prime \prime}(x)+\frac{\alpha}{x} u^{\prime}(x)=\delta x^{\delta-2}\left[\alpha+\delta-1+\delta x^{\delta}\right] u(x), x \in(0,1)$

with the boundary conditions $u(0)=1$ and $u(1)=e$.

\section{IADM solution}

Using the recurrence relation given by (89) and the same procedure as in examples above.

$$
u_{0}=u(0)+\frac{x-0}{1-0}[u(1)-u(0)]+L_{x x}^{-1}(r(x))
$$

With

$t(x)=\frac{\alpha}{x}, s(x)=-\delta x^{\delta-2}\left[\alpha+\delta-1+\delta x^{\delta}\right]$ and $r(x)=0$

and hence substituting the given constants $\alpha=\frac{1}{2}$ and $\delta=1$, in these equations one can easily get

$$
t=\frac{1}{2 x} \text { and } s=-\frac{1}{x}\left(\frac{1}{2}+x\right)
$$

Using these expressions the first component of the decomposition of the solution $u(x)$ can be found to be:

$$
u_{0}=1+(e-1) x
$$

Similar procedure as in previous examples the next four approximate solutions shown below are obtained with the help of MATHEMATICA using the recurrence relation (89)$$
\text { (2) }
$$ 


$$
\begin{aligned}
& u_{3}=-\frac{79 x}{360}-\left(-\frac{551543}{604800}+\frac{267917 \mathrm{e}}{604800}\right) x+\frac{221 \mathrm{e} x}{1440}-\frac{1741 x^{2}}{2880}+\frac{791 \mathrm{e} x^{2}}{2880}-\frac{53 x^{3}}{360}+\frac{191 \mathrm{e} x^{3}}{4320}+\frac{167 x^{4}}{3456}-\frac{3 \mathrm{e} x^{4}}{128}+\frac{53 x^{5}}{4800} \\
& -\frac{67 \mathrm{e} x^{5}}{14400}-\frac{7 x^{6}}{21600}-\frac{23 \mathrm{e} x^{6}}{21600}+\frac{x^{7}}{5040}-\frac{\mathrm{e} x^{7}}{5040}+\frac{79}{360} x \ln x-\frac{221 \mathrm{e} x \ln x}{1440}+\frac{31}{96} x^{2} \ln x-\frac{5}{48} \mathrm{e} x^{2} \ln x \\
& +\frac{7}{72} x^{3} \ln x-\frac{1}{96} \mathrm{e} x^{3} \ln x-\frac{1}{36} x^{4} \ln x+\frac{1}{72} \mathrm{e} x^{4} \ln x-\frac{1}{120} x^{5} \ln x+\frac{1}{240} \mathrm{e} x^{5} \ln x-\frac{1}{96} x(\ln x)^{2} \\
& -\frac{5}{96} \mathrm{e} x(\ln x)^{2}-\frac{1}{16} x^{2}(\ln x)^{2}+\frac{1}{32} \mathrm{e} x^{2}(\ln x)^{2}-\frac{1}{24} x^{3}(\ln x)^{2}+\frac{1}{48} \mathrm{e} x^{3}(\ln x)^{2}+\frac{1}{24} x(\ln x)^{3} \\
& -\frac{1}{48} \mathrm{e} x(\ln x)^{3} \\
& u_{4}=\frac{418823 x}{1209600}-\left(\frac{133427837}{169344000}-\frac{1732427 \mathrm{e}}{5080320}\right) x-\frac{175097 \mathrm{e} x}{1209600}+\frac{1187213 x^{2}}{2419200}-\frac{538187 \mathrm{e} x^{2}}{2419200}+\frac{168503 x^{3}}{3628800}-\frac{10813 \mathrm{e} x^{3}}{907200}-\frac{18193 x^{4}}{207360} \\
& +\frac{5047 \mathrm{e} x^{4}}{138240}-\frac{35611 x^{5}}{3456000}+\frac{9443 \mathrm{e} x^{5}}{3456000}+\frac{479 x^{6}}{216000}-\frac{307 \mathrm{e} x^{6}}{324000}+\frac{193 x^{7}}{635040}-\frac{437 \mathrm{e} x^{7}}{3175200}-\frac{17 x^{8}}{4233600}-\frac{11 \mathrm{e} x^{8}}{529200}+\frac{x^{9}}{362880} \\
& -\frac{\mathrm{e} x^{9}}{362880}-\frac{418823 x \ln x}{1209600}+\frac{175097 \mathrm{e} x \ln x}{1209600}-\frac{299 x^{2} \ln x}{5760}+\frac{169 \mathrm{e} x^{2} \ln x}{5760}+\frac{359 x^{3} \ln x}{5760}-\frac{59 \mathrm{e} x^{3} \ln x}{2160}+\frac{151 x^{4} \ln x}{3456} \\
& -\frac{107 \mathrm{e} x^{4} \ln x}{6912}+\frac{17 x^{5} \ln x}{2400}-\frac{47 \mathrm{e} x^{5} \ln x}{28800}-\frac{23 x^{6} \ln x}{21600}+\frac{23 \mathrm{e} x^{6} \ln x}{43200}-\frac{x^{7} \ln x}{5040}+\frac{\mathrm{e} x^{7} \ln x}{10080}-\frac{79 x(\ln x)^{2}}{1440}+\frac{221 \mathrm{e} x(\ln x)^{2}}{5760} \\
& -\frac{7}{384} x^{2}(\ln x)^{2}-\frac{1}{192} \mathrm{e} x^{2}(\ln x)^{2}-\frac{1}{72} x^{3}(\ln x)^{2}-\frac{1}{384} \mathrm{e} x^{3}(\ln x)^{2}-\frac{1}{144} x^{4}(\ln x)^{2}+\frac{1}{288} \mathrm{e} x^{4}(\ln x)^{2} \\
& -\frac{1}{480} x^{5}(\ln x)^{2}+\frac{1}{960} \mathrm{e} x^{5}(\ln x)^{2}+\frac{1}{576} x(\ln x)^{3}+\frac{5}{576} \mathrm{e} x(\ln x)^{3}+\frac{1}{96} x^{2}(\ln x)^{3}-\frac{1}{192} \mathrm{e} x^{2}(\ln x)^{3}+\frac{1}{144} x^{3}(\ln x)^{3} \\
& -\frac{1}{288} \mathrm{e} x^{3}(\ln x)^{3}-\frac{1}{192} x(\ln x)^{4}+\frac{1}{384} \mathrm{e} x(\ln x)^{4}
\end{aligned}
$$

\section{ADM solution}

Here the ADM is supported by the Maclaurin series expansion of functions to get in to the desired solution. The equation can be rewritten as $\left(x^{\alpha} u^{\prime}\right)^{\prime}=\delta x^{\alpha+\delta-2}\left[\alpha+\delta-1+\delta x^{\delta}\right] u(x)$

Using the scheme in (49),

$$
\begin{gathered}
u_{0}=1 \\
u_{n+1}=\delta \int_{0}^{x}\left(x^{-\alpha} \int_{0}^{x}\left[(\alpha+\delta-1) x^{\alpha+\delta-2}+\delta x^{\alpha+2 \delta-2}\right] u_{n} d x\right) d x
\end{gathered}
$$

From the recurrence relation (124), one can easily obtain

$$
\begin{gathered}
u_{0}=1 \\
u_{1}=x^{\delta}+\frac{\delta}{2(\alpha+2 \delta-1)} x^{2 \delta} \\
u_{2}=\frac{(\alpha+\delta-1)}{2(\alpha+2 \delta-1)} x^{2 \delta}+\frac{\delta(3 \alpha+5 \delta-3)}{6(\alpha+2 \delta-1)} x^{3 \delta}+\frac{\beta^{2}}{8(\alpha+2 \delta-1)(\alpha+4 \delta-1)} x^{4 \delta} \\
u_{3}=\frac{(\alpha+\delta-1)^{2}}{6(\alpha+2 \delta-1)(\alpha+3 \delta-1)} x^{3 \delta}+\frac{\delta(\alpha+\delta-1)(3 \alpha+7 \delta-3)}{12(\alpha+2 \delta-1)(\alpha+3 \delta-1)(\alpha+4 \delta-1)} x^{4 \delta} \\
+\frac{\beta\{3(\alpha+\delta-1)(\alpha+3 \delta-1)+4(3 \alpha+5 \delta-3)(\alpha+4 \delta-1)\}}{120(\alpha+2 \delta-1)(\alpha+3 \delta-1)(\alpha+4 \delta-1)(\alpha+5 \delta-1)} x^{5 \delta}+\frac{\delta^{3}}{48(\alpha+2 \delta-1)(\alpha+4 \delta-1)(\alpha+6 \delta-1)} x^{6 \delta}
\end{gathered}
$$

To show the possibility of obtaining the exact solution of the given equation, the approximate solution can be established by (56) the following way.

$$
\begin{gathered}
\Phi_{3}=u_{0}+u_{1}+u_{2} \\
=1+x^{\delta}+\frac{\delta}{2(\alpha+2 \delta-1)} x^{2 \delta}+\frac{(\alpha+\delta-1)}{2(\alpha+2 \delta-1)} x^{2 \delta}+\frac{\delta(3 \alpha+5 \delta-3)}{6(\alpha+2 \delta-1)} x^{3 \delta}+\frac{\beta^{2}}{8(\alpha+2 \delta-1)(\alpha+4 \delta-1)} x^{4 \delta} \\
\Phi_{4}=u_{0}+u_{1}+u_{2}+u_{3}
\end{gathered}
$$




$$
\begin{gathered}
=1+x^{\delta}+\frac{\delta}{2(\alpha+2 \delta-1)} x^{2 \delta}+\frac{(\alpha+\delta-1)}{2(\alpha+2 \delta-1)} x^{2 \delta}+\frac{\delta(3 \alpha+5 \delta-3)}{6(\alpha+2 \delta-1)} x^{3 \delta}+ \\
\frac{\beta^{2}}{8(\alpha+2 \delta-1)(\alpha+4 \delta-1)} x^{4 \delta}+\frac{(\alpha+\delta-1)^{2}}{6(\alpha+2 \delta-1)(\alpha+3 \delta-1)} x^{3 \delta}+\frac{\delta(\alpha+\delta-1)(3 \alpha+7 \delta-3)}{12(\alpha+2 \delta-1)(\alpha+3 \delta-1)(\alpha+4 \delta-1)} x^{4 \delta}+ \\
\frac{\beta\{3(\alpha+\delta-1)(\alpha+3 \delta-1)+4(3 \alpha+5 \delta-3)(\alpha+4 \delta-1)\}}{120(\alpha+2 \delta-1)(\alpha+3 \delta-1)(\alpha+4 \delta-1)(\alpha+5 \delta-1)} x^{5 \delta}+\frac{\delta^{3}}{48(\alpha+2 \delta-1)(\alpha+4 \delta-1)(\alpha+6 \delta-1)} x^{6 \delta} .
\end{gathered}
$$

By investigating the approximate solutions $\Phi_{2}, \Phi_{3}$ and $\Phi_{4}$, it can be observed that they agree with the Maclaurin expansion of the function $e^{x^{\delta}}$ up to $x^{\delta}, x^{2 \delta}$ and $x^{3 \delta}$ respectively. So, by evaluating more terms of the decomposition series, the exact solution is found to be

$$
u(x)=e^{x^{\delta}} .
$$

The following (figure 6) is plot by MATHEMATICA where the IADM solutions obtained using only the first four components of the decomposition solutions and the exact solution (for $\alpha=0.5, \delta=1$ ).

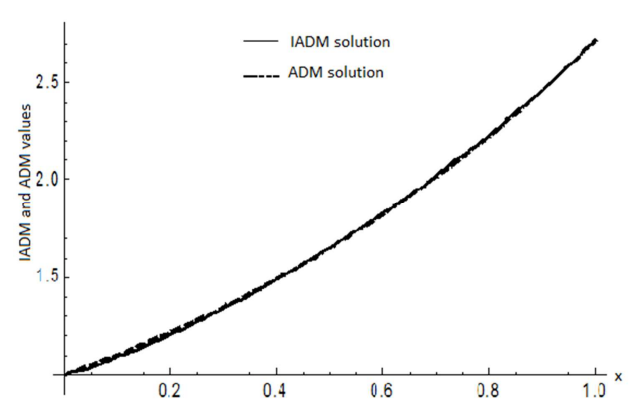

Figure 6. Comparison of the IADM and ADM solutions for example 5.2.

Table 2 shows the numerical illustration using the IADM and exact solutions for the problem. Unlike the figure, the first seven terms of the decomposition solution are used.

Table 2. Numerical results for example 5.2.

\begin{tabular}{llll}
\hline $\mathbf{x}$ & $\begin{array}{l}\text { Approximate Solution } \\
\boldsymbol{\Phi}_{\text {IADM }}\end{array}$ & $\begin{array}{l}\text { Exact Solution } \\
\boldsymbol{\Phi}_{\text {ADM }}\end{array}$ & $\begin{array}{l}\text { Error } / \boldsymbol{\Phi}_{A D M}- \\
\boldsymbol{\Phi}_{I A D M} /\end{array}$ \\
\hline 0 & 1 & 1 & 0 \\
0.2 & 1.221155823 & 1.221402758 & $2.47 \mathrm{E}-04$ \\
0.4 & 1.491930426 & 1.491824698 & $1.06 \mathrm{E}-04$ \\
0.6 & 1.822019687 & 1.8221188 & $9.91 \mathrm{E}-05$ \\
0.8 & 2.2256288 & 2.225540928 & $8.79 \mathrm{E}-05$ \\
1 & 2.718281828 & 2.718281828 & 0 \\
\hline
\end{tabular}

To verify how close the approximate solution is to the exact one: $u(x)=e^{x}$, the IADM solution considering the first four approximate results and the exact solution are plotted in figure 6 for $\alpha=0.5$ and $\delta=1$. It is shown from this figure that the approximate solution obtained through the IADM is very close to the exact one using very few terms. Furthermore, numerical results are shown in the table using the first seven IADM solutions of the example considered. From the absolute error in the table it is observed that the IADM solution is closer to the exact solution.. Lin, Y. and Chen, C. K. used a three-point finite difference method in which a huge amount of computational work is needed to obtain the numerical solution [14].

Example 5.2. Consider the nonlinear boundary value problem

$u^{\prime \prime}-\frac{1}{x} u^{\prime}-\frac{x^{2}}{3} u^{5}=0$ with $u(0)=1$ and $u^{\prime}(1)=-\frac{\sqrt{3}}{8}$.

\section{MADM solution}

To treat second order BVPs with mixed boundary conditions of this form, the operators at (67) and (69) can be reduced, respectively, to

and

$$
L_{1}=x^{2-k} \frac{d}{d x}\left(x^{k} \frac{d}{d x}\right)
$$

$$
L_{1}^{-1}=\int_{0}^{x} x^{-k} \int_{b}^{x} x^{k-2}(.) d x d x
$$

Hence, from (106) and (107) one can define the operators below to treat the problem considered.

$$
L_{1}=x^{3} \frac{d}{d x}\left(x^{-1} \frac{d}{d x}\right)
$$

and

$$
L_{1}^{-1}=\int_{0}^{x} x \int_{1}^{x} x^{-3}(.) d x d x
$$

Rearranging and multiplying the given equation $u^{\prime \prime}-$ $\frac{1}{x} u^{\prime}-\frac{x^{2}}{3} u^{5}=0$ by $x^{2}$, in an operator form it becomes

$$
L_{1} u=\frac{x^{4}}{3} u^{5}
$$

Applying (128) on both sides of (129) one can get

$$
u(x)=u(0)+\frac{1}{2} u^{\prime}(1) x^{2}+L_{1}^{-1} \frac{x^{4}}{3} u^{5}
$$

Using the decomposition series for the linear function $u(x)$ and the Adomian polynomial series for the nonlinear term $u^{5}$, the following expression can be obtained.

$$
\sum_{n=0}^{\infty} u_{n}=u(0)+\frac{1}{2} u^{\prime}(1) x^{2}+L_{1}^{-1}\left(\frac{x^{4}}{3} \sum_{n=0}^{\infty} A_{n}\right)
$$

This gives the recursive relationship

$$
\begin{aligned}
& u_{0}(x)=u(0)+\frac{1}{2} u^{\prime}(1) x^{2} \\
& u_{n+1}(x)=L_{1}^{-1}\left(\frac{x^{4}}{3} A_{n}\right), n \geq 0 .
\end{aligned}
$$

Now to determine the Adomian polynomials for the nonlinear term, call it $p(u)=u^{5}$, the Adomian formula given by (3) is used in (132) as follows.

For $n=0$, 


$$
\begin{gathered}
A_{0}=p\left(u_{0}\right) \\
\Rightarrow A_{0}=u_{0}^{5} \\
\text { For } n=1, \\
A_{1}=\frac{d}{d \lambda}\left[p\left(u_{0}+\lambda u_{1}\right)\right]_{\lambda=0} \\
\Rightarrow A_{1}=5 u_{0}^{4} u_{1}
\end{gathered}
$$

For $n=2,3, \ldots$ the respective Adomian polynomials can be obtained in similar fashion.

Or, optionally, MATHEMATICA is set to give desired number of decomposition terms of any nonlinearity types as shown in the appendix C. For instance,

For $n=2$,

MATHEMATICA displayed the corresponding Adomian polynomials in terms of the subscript of the term $u$ for $A_{2}$ as

$$
\begin{gathered}
\mathrm{u}[2] F^{\prime}[0]+\frac{1}{2} u[1]^{2} F^{\prime \prime}[0] \\
u_{0}=1-\frac{\sqrt{3} x^{2}}{16} \\
u_{1}=-\frac{1}{176160768 \sqrt{3}} x^{2}\left(-6961983+15255520 \sqrt{3}-7340032 \sqrt{3} x^{2}+2293760 x^{4}-143360 \sqrt{3} x^{6}+16128 x^{8}-\right. \\
\left.336 \sqrt{3} x^{10}+9 x^{12}\right) \\
u_{2}=-\frac{1}{831230790598656 \sqrt{3}} 5\left(\left(\frac{4274979839517}{2}-2274393702976 \sqrt{3}\right) x^{2}+458752(-994569+2179360 \sqrt{3}) x^{6}-\right. \\
286720(1307616+639947 \sqrt{3}) x^{8}+5376(26731043+3922848 \sqrt{3}) x^{10}-448(3922848+35474273 \sqrt{3}) x^{12}+ \\
3(1087535333+6538080 \sqrt{3}) x^{14}-155713536 \sqrt{3} x^{16}+16220160 x^{18}-405504 \sqrt{3} x^{20}+20736 x^{22}-216 \sqrt{3} x^{24}+ \\
\left.\frac{81 x^{26}}{26}\right)
\end{gathered}
$$

The approximate solution of the nonlinear homogeneous boundary value problem considering only the first three iterative results is thus approximately given using (56) by:

$$
\Phi_{3}=u_{0}+u_{1}+u_{2}
$$

\section{Exact solution}

The exact solution of the nonlinear BVP is given by Kim, W. and Chun, C. is [12]:

$$
u(x)=\frac{1}{\sqrt{1+\frac{x^{2}}{3}}}
$$

The graphs of the example from the MADM and exact solutions are plotted using MATHEMATICA in figure 7. this means

$$
A_{2}=u_{2} f^{\prime}\left(u_{0}\right)+\frac{1}{2} u_{1}^{2} f^{\prime \prime}\left(u_{0}\right)
$$

where $f$ in this case is $p$. Hence, only by substituting the appropriate derivatives of the nonlinear function $p$ the desired result follows for $A_{2}$ as

$$
A_{2}=5 u_{2} u_{0}^{4}+10 u_{1}^{2} u_{0}^{3}
$$

The same procedure gives the rest of the polynomials:

$$
A_{3}=5 u_{3} u_{0}^{4}+20 u_{2} u_{1} u_{0}^{3}+10 u_{1}^{3} u_{0}^{2}
$$

Substituting these results of the Adomian polynomials in to (132) gives the components 
Table 3. Numerical results for example 5.3.

\begin{tabular}{llll}
\hline $\mathbf{x}$ & $\begin{array}{l}\text { MADM solution } \\
\boldsymbol{\Phi}_{\text {MADM }}\end{array}$ & $\begin{array}{l}\text { Exact } \\
\text { Solution }\end{array}$ & /Exact solution- $\boldsymbol{\Phi}_{\boldsymbol{M A D M}} /$ \\
\hline 0 & 1 & 1 & 0 \\
0.1 & 0.998335705 & 0.998337488 & $1.7830 \mathrm{E}-06$ \\
0.2 & 0.993392139 & 0.993399268 & $7.1290 \mathrm{E}-06$ \\
0.3 & 0.985313275 & 0.985329278 & $1.6003 \mathrm{E}-05$ \\
0.4 & 0.974326442 & 0.974354704 & $2.8262 \mathrm{E}-05$ \\
0.5 & 0.960725402 & 0.960768923 & $4.3521 \mathrm{E}-05$ \\
0.6 & 0.944850222 & 0.944911183 & $6.0961 \mathrm{E}-05$ \\
0.7 & 0.92706639 & 0.927145541 & $7.9151 \mathrm{E}-05$ \\
0.8 & 0.907745218 & 0.907841299 & $9.6081 \mathrm{E}-05$ \\
0.9 & 0.887246455 & 0.887356509 & $1.1005 \mathrm{E}-04$ \\
1 & 0.865920000 & 0.866025404 & $1.0540 \mathrm{E}-04$ \\
\hline
\end{tabular}

From the example above it can easily be observed that the modification of the decomposition method MADM can easily treat problems that the standard ADM fails to be effective, even resulting in a good agreement with the exact solution. Only the first three iterative results of the solution are used to plot the graph. Overlapping of the graphs implies the results obtained by MADM are as accurate as the results from the exact solution. In addition, numerical results are shown in table 3 considering only the first four iterative results of the approximate solution in order to get better numerical result. The absolute error determined show that the method is too accurate to treat nonlinear singular two-point boundary value problems with mixed boundary conditions. Example 5.3. Consider the nonlinear singular BVP

$$
u^{\prime \prime}(x)+\frac{1}{2 x} u^{\prime}(x)=e^{u}\left(\frac{1}{2}-e^{u}\right), x \in(0,1)
$$

subject to the boundary conditions $u(0)=\ln (2)$ and $u(1)=0$.

\section{IADM solution}

As can be expected, it is necessary to represent the nonlinear part by the Adomian polynomials. Here the nonlinear term is

$$
t(u)=e^{u}\left(0.5-e^{u}\right)
$$

The required recurrence relation is (90) which is given by:

$$
\begin{gathered}
u_{0}(x)=u(a)+\frac{x-a}{b-a}[u(b)-u(a)]+L^{-1}{ }_{x x}[r(x)] \\
u_{n+1}(x)=-L_{x x}^{-1}\left[t(x) u_{n}^{\prime}(x)+s(x) A_{n}(x)\right], n \geq 0 \\
\Rightarrow u_{0}=\ln 2+x(-\ln 2)=(1-x) \ln 2
\end{gathered}
$$

But for fast convergence to the exact solution, MADM by Wazwaz, A.-M. helps to rearrange the result obtained above so that $u_{0}$ assumes to be zero and all the existing terms obtained to be added to $u_{1}$ the following way [21]:

For $n=0, u_{1}=-L_{x x}^{-1}\left[\frac{1}{2 x} u_{n}^{\prime}+A_{0}\right]$

The Adomian polynomial $A_{0}$ can be determined either using the Adomian formula (3) or the results provided by MATHEMATICA at appendix $\mathrm{C}$ to be:

$$
\begin{aligned}
& A_{0}=t\left(u_{0}\right)=e^{u_{0}}\left(0.5-e^{u_{0}}\right) \\
& \Longrightarrow L^{-1}{ }_{x x}[-0.5]=\frac{1}{4} x-\frac{1}{4} x^{2}
\end{aligned}
$$

Adding the previous result of $u_{0}$ i.e. $(1-x) \ln 2$ to (134) one can get $u_{1}$ as:

$$
u_{1}=(1-x) \ln 2+\frac{1}{4} x-\frac{1}{4} x^{2}
$$

Recall that $A_{1}=u_{1} t^{\prime}\left(u_{0}\right)$

$$
\begin{gathered}
A_{2}=u_{2} t^{\prime}\left(u_{0}\right)+\frac{1}{2} u_{1}^{2} t^{\prime \prime}\left(u_{0}\right) \\
A_{3}=u_{3} t^{\prime}\left(u_{0}\right)+u_{1} u_{2} t^{\prime \prime}\left(u_{0}\right)+\frac{1}{6} u_{1}^{3} t^{\prime \prime \prime}\left(u_{0}\right) \\
\text { i. e. }, A_{1}=\frac{1}{2}\left(1-4 e^{u_{0}}\right) u_{1} \\
A_{2}=\frac{1}{4} e^{u_{0}}\left[u_{1}^{2}+2 u_{2}-8 e^{u_{0}}\left(u_{1}^{2}+u_{2}\right)\right] \\
A_{3}=\frac{1}{12} e^{u_{0}}\left[u_{1}^{3}+6 u_{1} u_{2}+6 u_{3}-8 e^{u_{0}}\left(2 u_{1}^{3}+6 u_{1} u_{2}\right.\right. \\
\left.\left.+3 u_{3}\right)\right]
\end{gathered}
$$

For $n=1$,

$u_{2}$ from the second equation of the recurrence relation would be:

$$
\begin{aligned}
& u_{2}=-L_{x x}^{-1}[ \frac{1}{2 x}\left(\frac{1}{4} \ln 2-\frac{1}{2} x\right)+\frac{3}{2}\left((1-x) \ln 2+\frac{1}{4} x\right. \\
&\left.\left.-\frac{1}{4} x^{2}\right)\right] \\
& \Rightarrow u_{2}=\frac{x}{8}+\frac{x^{2}}{8}-\frac{x^{3}}{16}+\frac{x^{4}}{32}-\frac{3}{4} x^{2} \ln 2 \\
&+x\left(-\frac{7}{32}+\frac{3 \ln 2}{4}+\frac{\ln 16}{16}\right)-\frac{1}{8} x \ln 16+ \\
& \frac{1}{16} x^{3} \ln 16-\frac{1}{8} x \ln x+\frac{1}{8} x \ln 16 \ln x
\end{aligned}
$$

The terms of the decomposition components are getting too vast to solve by hand but MATHEMATICA facilitates computing. So it can be used to list as many decomposition terms as desired. Though more than ten terms of the decomposition are used in the IADM solution, it is believed not economical to write the next iterative results, it is found important to list only up to the fifth iteration below.

$$
\begin{gathered}
u_{3}=-\frac{3 x}{64}-\frac{x^{2}}{16}+\frac{5 x^{3}}{384}-\frac{23 x^{4}}{768}+\frac{x^{5}}{64}-\frac{x^{6}}{192}+\frac{3}{8} x \ln 2+\frac{3}{8} x^{2} \ln 2-\frac{1}{3} x^{3} \ln 2+\frac{23}{96} x^{4} \ln 2-\frac{7}{160} x^{5} \ln 2-\frac{7}{8} x^{2}(\ln 2)^{2}+\frac{7}{12} x^{3}(\ln 2)^{2}- \\
\frac{7}{48} x^{4}(\ln 2)^{2}-\frac{1}{16} x \ln 16+\frac{5}{192} x^{3} \ln 16-\frac{3}{640} x^{5} \ln 16+x\left(\frac{89}{768}-\frac{49 \ln 2}{80}+\frac{7(\ln 2)^{2}}{16}+\frac{79 \ln 16}{1920}-\frac{\ln 256}{64}\right)+\frac{1}{64} x \ln 256+\frac{3}{64} x \ln x+\frac{1}{32} x^{3} \ln x- \\
\frac{3}{8} x \ln 2 \ln x+\frac{1}{16} x \ln 16 \ln x-\frac{1}{32} x^{3} \ln 16 \ln x-\frac{1}{64} x \ln 256 \ln x+\frac{1}{32} x(\ln x)^{2}-\frac{1}{32} x \ln 16(\ln x)^{2} \\
u_{4}=\frac{1}{7680} x\left(-345+912 \ln 2-1976(\ln 2)^{2}+\frac{1}{7}(210-524 \ln 2-79 \ln 16)-\frac{3}{2}\left(-51-1616(\ln 2)^{2}+320(\ln 2)^{3}-56 \ln 2(-15+\right.\right.
\end{gathered}
$$




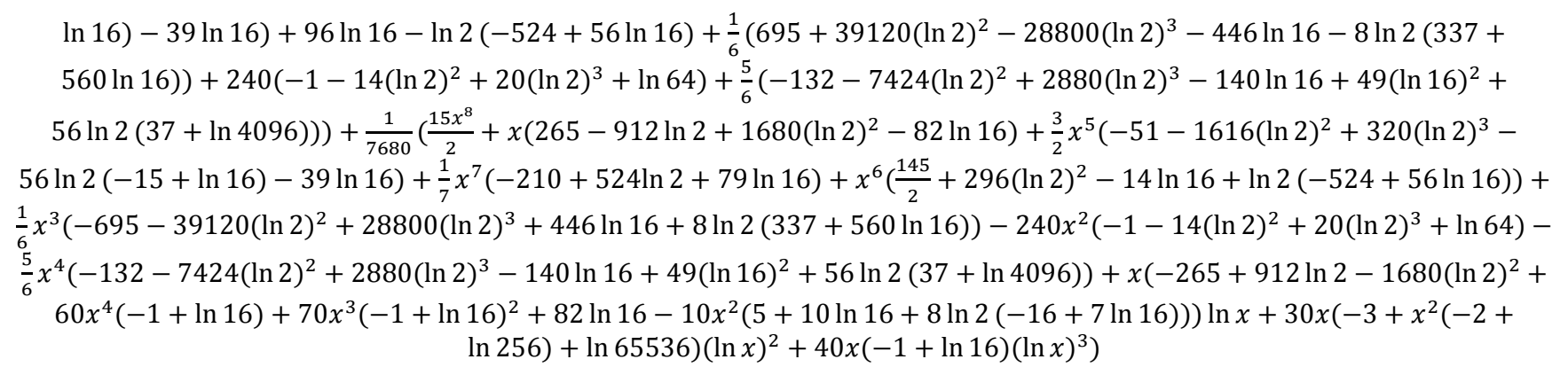

Hence, the result obtained by (71) is used to show the numerical illustrations in table 4.

II. Exact solution

Consider the given equation

$$
u^{\prime \prime}(x)+\frac{1}{2 x} u^{\prime}(x)=e^{u}\left(\frac{1}{2}-e^{u}\right), x \in(0,1)
$$

Re writing it in the form of (41) and using (56) one can get:

$$
\begin{gathered}
u_{0}=u(0) \\
u_{n+1}=\int_{0}^{x}\left(x^{-\alpha} \int_{0}^{x}\left[r(x) A_{n}\right] d x\right) d x, n \geq 0
\end{gathered}
$$

Here $\alpha=\frac{1}{2}$ and $r(x)=x^{1 / 2}$ but the Adomian polynomials are already determined in IADM solution above.

From the recursive relation above it can be obtained that

$$
u_{0}=\ln (2)
$$

For $n=0$, (135) reduces to

$$
\begin{gathered}
u_{1}=\int_{0}^{x}\left(x^{-1 / 2} \int_{0}^{x}\left[x^{1 / 2} e^{u_{0}}\left(0.5-e^{u_{0}}\right)\right] d x\right) d x \\
=-x^{2}
\end{gathered}
$$

Similarly using (135) one can easily observe that

$$
u_{2}=\frac{x^{4}}{2}
$$

$$
\begin{gathered}
u_{3}=-\frac{x^{6}}{3} \\
u_{4}=\frac{x^{8}}{4} \\
u_{5}=-\frac{x^{10}}{5}
\end{gathered}
$$

$$
u_{n}=(-1)^{n} \frac{x^{2 n}}{n}, n \geq 1(136)
$$

Now these results by the ADM can be used to get the series expansion of $\ln \left(1+x^{2}\right)$. The solution is then given by

$$
u=\sum_{n=0}^{\infty} u_{n}(137)
$$

$$
\begin{aligned}
& =\ln (2)+\sum_{n=1}^{\infty} \frac{(-1)^{n} x^{2 n}}{n} \\
& \Rightarrow u(x)=\ln \left(\frac{2}{1+x^{2}}\right)
\end{aligned}
$$

which is the exact solution.

MATLAB is used to plot the IADM and exact solutions in the figure 8 .



Figure 8. Comparison of the IADM and exact solutions for example 5.4. 
The numerical illustrations of the problem considered are shown in table below.

Table 4. Numerical results for example 5.4.

\begin{tabular}{llll}
\hline $\mathbf{x}$ & $\begin{array}{l}\text { Approximate } \\
\text { solution } \boldsymbol{\Phi}_{\text {IADM }}\end{array}$ & $\begin{array}{l}\text { Exact solution } \\
\boldsymbol{u}(\boldsymbol{x})\end{array}$ & $\begin{array}{l}\text { Error } / \boldsymbol{u}(\boldsymbol{x})- \\
\boldsymbol{\Phi}_{\text {IADM }} /\end{array}$ \\
\hline 0 & 0.693147181 & 0.693147181 & 0 \\
0.1 & 0.683195177 & 0.683196850 & $1.67 \mathrm{E}-06$ \\
0.2 & 0.653924627 & 0.653926467 & $1.84 \mathrm{E}-06$ \\
0.3 & 0.606967226 & 0.606969484 & $2.26 \mathrm{E}-06$ \\
0.4 & 0.544725404 & 0.544727175 & $1.77 \mathrm{E}-06$ \\
0.5 & 0.470002399 & 0.470003629 & $1.23 \mathrm{E}-06$ \\
0.6 & 0.385661666 & 0.385662481 & $8.15 \mathrm{E}-07$ \\
0.7 & 0.294370545 & 0.294371061 & $5.16 \mathrm{E}-07$ \\
0.8 & 0.198450643 & 0.198450939 & $2.96 \mathrm{E}-07$ \\
0.9 & 0.099820206 & 0.099820335 & $1.29 \mathrm{E}-07$ \\
1 & 0 & 0 & 0 \\
\hline
\end{tabular}

In the overlapping plots shown above, one can easily observe that the IADM and the exact solutions are nearly identical. Furthermore, numerical results are shown in table 4 in which an absolute error $\leq 10^{-6}$ is obtained. This shows that the IADM converges faster to the exact solution. Unlike that of the DTM, in this example it can easily be observed that it is not difficult to obtain exact solutions for nonlinear inhomogeneous BVPs using the standrad ADM.

\section{Summary, Conclusion and Recommendations}

\subsection{Summary}

Convective triangular fins with invariant and power-law temperature-dependent thermal conductivities were studied by MADM. Through MATLAB and MATHEMATICA, reliable parametric solutions for both problems was offered by the help of the modification. A realistic model problem regarding a silicon fin of specific dimensions was carried out as a numerical illustration. The MADM solutions were compared with an exact analytical solution by Arpaci, S. Vedat for the constant $k$ (governed by linear BVP) and a numerical solution via FDM by Mokheimer Esmail, M. A for power-law temperature-dependent $k$ cases (governed by nonlinear BVP) [2], [17]. Moreover, it was observed that $\mathrm{ADM}$ and its modifications; MADM and IADM are comfortable to treat the problems. The approximate solutions obtained by MADM and IADM converge faster to the respective exact solutions. Some numerical approximations by IADM are compared with the results in some other methods like reproducing kernel space method by Cui, M. and Geng, $\mathrm{F}$ in which magnificent power of fast convergent behavior of IADM is observed [4].

Further more, this research also considered second order STPBVPs both numerically and analytically using the standard and improved ADMs. The results were compared by plotting the graphs of the approximate and exact solutions on the same coordinate plane by using the symbolic softwares
MATLAB and MATHEMATICA. Error analysis was performed to see how close the approximate solutions to the exact solutions are.

\subsection{Conclusion}

Temperature distribution with constant and variable thermal conductivity along triangular fin profile governed respectively by linear and nonlinear two-point BVPs can be treated directly using the improved ADMs.

Thermal equations of linear and nonlinear BVPs can be treated directly through the standard ADM and its modifications effectively.

Although the classical ADM is very powerful, it fails in treating some singular boundary value problems due to the existence of singular point at $x=0$. So this difficulty is alleviated by the modifications MADM and IADM; and shown by treating STPBVPs with Dirchlet and mixed boundary conditions holding singular feature both numerically and analytically.

Though the methods are very convenient for software treatments, it is noted that the modified decomposition methods (especially IADM) encounters difficulties in obtaining each component for some complex nonlinear problems even if symbolic packages are used since each component is obtained by cumbersome definite integrals.

\subsection{Recommendations}

Based on the analysis of thermal equations via the improved Adomian decomposition methods the following basic recommendations are suggested:

1) To study heat transfer from extended surfaces it is undisputable to study the temperature distribution along the fins length. So individuals who are interested to work on heat transfer in these areas can benefit from the results and the MATLAB codes presented.

2) Two-point BVPs with single singular point were treated by the modifications. It would be worthwhile to expand application of the operators so that the method could be used to treat two-point BVPs with multiple singularities.

3) The ADM is not only used in a straight forward manner but also requires less computational works in comparison to some other methods like HAM and MVIM. To the contrary, though the series can be rapidly convergent in a very small region, it has very slow convergence rate in wider regions and the truncated series solution is an inaccurate solution in that region which will seriously restrict the application area of the method. An investigation into this claim would greatly benefit the scientific community.

\section{Acknowledgements}

I respect all the authors whose works played an important role in the success of this research work. 
I appreciate all SciencePG editorial team for the thorough

evaluation and valuable comments.

\section{Appendix}

\section{Appendix A. MATLAB Code to Get the Decomposition Terms of the Recurrence Relation (102).}

clear all \%clears previously declared symbols.

clc \%clears page.

syms L $\mathrm{m}$ x xx s Thetha_b \%syms declares the variables used to be symbolic.

nth=input('How many decomposition terms do you want to include in your solution? ');

$\%$ invites to insert the number of decomposition terms needed to be used in the decomposition.

$\mathrm{f}=1 ; \mathrm{s}=0$;

for $\mathrm{n}=1: \mathrm{nth}$

$\mathrm{s}=\mathrm{s}+\mathrm{f}$;

$\operatorname{disp}(\operatorname{sprintf}(' \%$ s\%d', 'Thetha_', n-1,'='))\%displays each component of the decomposition with \%their respective values.

$\operatorname{disp}\left(\mathrm{f}^{*}\right.$ Thetha_b)

$\mathrm{f}=\operatorname{int}\left((1 / \mathrm{xx}) * \operatorname{int}\left(\mathrm{m}^{\wedge} 2 * \mathrm{f}, \mathrm{x}, 0, \mathrm{xx}\right), \mathrm{xx}, \mathrm{L}, \mathrm{x}\right) ; \%$ MADM operator in the form int(expr,var,a,b); it \%computes definite integral.

end

solution $=\mathrm{S}$ *Thetha_b \%finally this displays the sum of the results from the for loop.

\section{Appendix B. MATLAB Codes to Get the Decomposition Terms of the Recurrence Relation (116).}

\section{For $\boldsymbol{A}_{k}$}

$\%$ Function $\mathrm{Ak}$, returning the $\mathrm{k}^{\text {th }}$ component of the Adomian polynomials corresponding to $\%$ the nonlinearity A.

$\%$ Beginning

function $\mathrm{Ak}=\mathrm{f}(\mathrm{k})$

syms x s h

sym('u0(x)');sym('u1(x)');sym('u2(x)');sym('u3(x)');sym('u4(x)');sym('u5(x)');sym('u6(x)');sym('u7(x)');sym('u8(x)');sym('u9( x)');sym('u10(x)');sym('u11(x)');sym('u12(x)');sym('u13');sym('u14');sym('u15');sym('u16'); sym('u17');sym('u18'); sym('u19'); $\operatorname{sym}(' \mathrm{u} 20$ ');

$\left.\left.\mathrm{s}={ }^{\prime} \mathrm{u} 0(\mathrm{x})^{\prime}+\mathrm{h}^{* \prime} \mathrm{u} 1(\mathrm{x})^{\prime}+\mathrm{h}^{\wedge} 2^{* \prime} \mathrm{u} 2(\mathrm{x})^{\prime}+\mathrm{h}^{\wedge} 3 *^{*} \mathrm{u} 3(\mathrm{x})^{\prime}+\mathrm{h}^{\wedge} 4{ }^{* \prime} \mathrm{u} 4(\mathrm{x})^{\prime}+\mathrm{h}^{\wedge} 5^{* \prime} \mathrm{u} 5(\mathrm{x})^{\prime}+\mathrm{h}^{\wedge} 6^{* \prime} \mathrm{u} 6(\mathrm{x})^{\prime}+\mathrm{h}^{\wedge} 7 *^{\prime} \mathrm{u} 7(\mathrm{x})^{\prime}+\mathrm{h}^{\wedge} 8^{* \prime} \mathrm{u} 8(\mathrm{x})\right)^{\prime}+\mathrm{h}^{\wedge} 9^{* \prime} \mathrm{u} 9(\mathrm{x})\right)^{\prime}+\mathrm{h}^{\wedge}$

$10^{* \prime} \mathrm{u} 10(\mathrm{x})^{\prime}+\mathrm{h}^{\wedge} 11^{* \prime} \mathrm{u} 11(\mathrm{x})^{\prime}+\mathrm{h}^{\wedge} 12^{* \prime} \mathrm{u} 12(\mathrm{X})^{\prime}+\mathrm{h}^{\wedge} 13^{* \prime} \mathrm{u} 13^{\prime}+\mathrm{h}^{\wedge} 14^{* \prime} \mathrm{u} 14^{\prime}+\mathrm{h}^{\wedge} 15^{* \prime} \mathrm{u} 15^{\prime}+\mathrm{h}^{\wedge} 16^{* \prime} \mathrm{u} 16^{\prime}+\mathrm{h}^{\wedge} 17^{* \prime} \mathrm{u} 17^{\prime}+\mathrm{h}^{\wedge} 18^{* \prime} \mathrm{u} 18^{\prime}+\mathrm{h}^{\wedge} 19^{* \prime} \mathrm{u} 19$

' $+\mathrm{h}^{\wedge} 20 * 1 \mathrm{u} 20$ ';

$\mathrm{Ak}=(1 / \text { factorial }(\mathrm{k}))^{*} \operatorname{subs}\left(\operatorname{diff}\left(1 / \mathrm{s} *(\operatorname{diff}(\mathrm{s}, \mathrm{x}))^{\wedge} 2, \mathrm{~h}, \mathrm{k}\right), \mathrm{h}, 0\right)$;

$\%$ The End

2. For $B_{k}$

$\%$ Function $\mathrm{Bk}$, returning the $\mathrm{k}^{\text {th }}$ component of the Adomian polynomials corresponding to $\%$ the nonlinearity $\mathrm{B}$.

$\%$ Beginning

function $\mathrm{Bk}=\mathrm{f}(\mathrm{k})$

syms x s h Z

sym('u0(x)');sym('u1(x)');sym('u2(x)');sym('u3(x)');sym('u4(x)');sym('u5(x)');sym('u6(x)');sym('u7(x)');sym('u8(x)');sym('u9( x)');sym('u10(x)');sym('u11(x)');sym('u12(x)');sym('u13');sym('u14');sym('u15');sym('u16'); sym('u17');sym('u18'); sym('u19'); sym('u20');

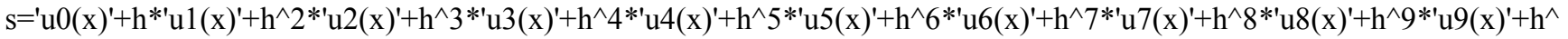
$10^{* \prime} \mathrm{u} 10(\mathrm{x})^{\prime}+\mathrm{h}^{\wedge} 11^{* \prime} \mathrm{u} 11(\mathrm{x})^{\prime}+\mathrm{h}^{\wedge} 12^{* \prime} \mathrm{u} 12(\mathrm{x})^{\prime}+\mathrm{h}^{\wedge} 13^{* \prime} \mathrm{u} 13^{\prime}+\mathrm{h}^{\wedge} 14^{* \prime} \mathrm{u} 14^{\prime}+\mathrm{h}^{\wedge} 15^{* \prime} \mathrm{u} 15^{\prime}+\mathrm{h}^{\wedge} 16^{* \prime} \mathrm{u} 16^{\prime}+\mathrm{h}^{\wedge} 17^{* \prime} \mathrm{u} 17^{\prime}+\mathrm{h}^{\wedge} 18^{* \prime} \mathrm{u} 18^{\prime}+\mathrm{h}^{\wedge} 19^{*} \mathrm{u} 19^{\prime}$ $+\mathrm{h}^{\wedge} 20 *{ }^{\prime \prime} \mathrm{u} 20^{\prime}$

$\mathrm{Bk}=(1 /$ factorial $(\mathrm{k})){ }^{*} \operatorname{subs}\left(\operatorname{diff}\left(\mathrm{s}^{\wedge}(1-\mathrm{Z}), \mathrm{h}, \mathrm{k}\right), \mathrm{h}, 0\right)$;

$\%$ The End

3. For $C_{k}$

$\%$ Function $\mathrm{Ck}$, returning the kth component of the Adomian polynomials corresponding to the \%nonlinearity $\mathrm{C}$.

$\%$ Beginning

function $\mathrm{Ck}=\mathrm{f}(\mathrm{k})$

syms x s h Z

sym('u0(x)'); sym('u1(x)');sym('u2(x)');sym('u3(x)'); sym('u4(x)');sym('u5(x)');sym('u6(x)');sym('u7(x)');sym('u8(x)');sym('u9( x)');sym('u10(x)');sym('u11(x)');sym('u12(x)');sym('u13');sym('u14');sym('u15');sym('u16'); sym('u17');sym('u18'); sym('u19'); sym('u20');

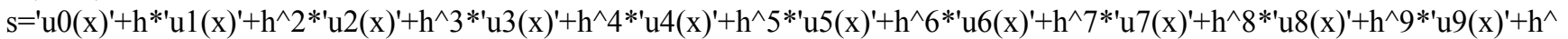
$10^{* \prime} \mathrm{u} 10(\mathrm{x})^{\prime}+\mathrm{h}^{\wedge} 11^{* \prime} \mathrm{u} 11(\mathrm{x})^{\prime}+\mathrm{h}^{\wedge} 12^{* \prime} \mathrm{u} 12(\mathrm{x})^{\prime}+\mathrm{h}^{\wedge} 13^{* \prime} \mathrm{u} 13^{\prime}+\mathrm{h}^{\wedge} 14^{* \prime} \mathrm{u} 14^{\prime}+\mathrm{h}^{\wedge} 15^{* \prime} \mathrm{u} 15^{\prime}+\mathrm{h}^{\wedge} 16^{* \prime} \mathrm{u} 16^{\prime}+\mathrm{h}^{\wedge} 17^{* \prime} \mathrm{u} 17^{\prime}+\mathrm{h}^{\wedge} 18^{* \prime} \mathrm{u} 18^{\prime}+\mathrm{h}^{\wedge} 19^{* \prime} \mathrm{u} 19^{\prime}$ 




\section{Appendix C. Decomposition of Adomian Polynomials Using MATHEMATICA}

The following is a MATHEMATICA program to get any number $n$ of the Adomian polynomials of any type of nonlinearity developed based on the subscripts of the term $u$.

AdomianPolynomials[u_, $\left.\mathrm{F}_{-}, \mathrm{o}_{-}\right]:=\operatorname{CoefficientList}\left[\operatorname{Expand} A l l\left[\operatorname{Series}\left[F\left[\operatorname{Sum}\left[\lambda^{\wedge} k u[k],\{k, o\}\right]\right],\{\lambda, 0, o\}\right]\right], \lambda\right]$

AdomianPolynomials $[u, F, n] / /$ ColumnForm

Based on the code, the first six terms of the Adomian polynomials in Wolfram language are displayed in the following way: 


$$
\begin{gathered}
\mathrm{u}[1] F^{\prime}[0] \\
\mathrm{u}[2] F^{\prime}[0]+\frac{1}{2} u[1]^{2} F^{\prime \prime}[0] \\
\mathrm{u}[3] F^{\prime}[0]+u[1] u[2] F^{\prime \prime}[0]+\frac{1}{6} u[1]^{3} F^{\prime \prime \prime}[0] \\
\mathrm{u}[4] F^{\prime}[0]+\frac{1}{2} u[2]^{2} F^{\prime \prime}[0]+u[1] u[3] F^{\prime \prime}[0]+\frac{1}{2} u[1]^{2} u[2] F^{\prime \prime \prime}[0]+\frac{1}{24} u[1]^{4} F^{(4)}[0] \\
\mathrm{u}[5] F^{\prime}[0]+u[2] u[3] F^{\prime \prime}[0]+u[1] u[4] F^{\prime \prime}[0]+\frac{1}{2} u[1] u[2]^{2} F^{\prime \prime \prime}[0]+\frac{1}{2} u[1]^{2} u[3] F^{\prime \prime \prime}[0] \\
+\frac{1}{6} u[1]^{3} u[2] F^{(4)}[0]+\frac{1}{120} u[1]^{5} F^{(5)}[0]
\end{gathered}
$$

These results displayed above by MATHEMATICA would generally mean respectively that

$$
\begin{gathered}
A_{0}=f\left(u_{0}\right) \\
A_{1}=u_{1} f^{\prime}\left(u_{0}\right) \\
A_{2}=u_{2} f^{\prime}\left(u_{0}\right)+\frac{1}{2} u_{1}^{2} f^{\prime \prime}\left(u_{0}\right) \\
A_{3}=u_{3} f^{\prime}\left(u_{0}\right)+u_{1} u_{2} f^{\prime \prime}\left(u_{0}\right)+\frac{1}{6} u_{1}^{3} f^{\prime \prime \prime}\left(u_{0}\right) \\
A_{4}=u_{4} f^{\prime}\left(u_{0}\right)+\frac{1}{2} u_{2}^{2} f^{\prime \prime}\left(u_{0}\right)+u_{1} u_{3} f^{\prime \prime}\left(u_{0}\right)+\frac{1}{2} u_{1}^{2} u_{2} f^{\prime \prime \prime}\left(u_{0}\right)+\frac{1}{24} u_{1}^{4} f^{(4)}\left(u_{0}\right) \\
A_{5}=u_{5} f^{\prime}\left(u_{0}\right)+u_{2} u_{3} f^{\prime \prime}\left(u_{0}\right)+u_{1} u_{4} f^{\prime \prime}\left(u_{0}\right)+\frac{1}{2} u_{1} u_{2}^{2} f^{\prime \prime \prime}\left(u_{0}\right)+\frac{1}{2} u_{1}^{2} u_{3} f^{\prime \prime \prime}\left(u_{0}\right)+\frac{1}{26} u_{1}^{3} u_{2} f^{(4)}\left(u_{0}\right)+\frac{1}{120} u_{1}^{5} f^{(5)}\left(u_{0}\right)
\end{gathered}
$$

\section{References}

[1] Adomian, G. 1988. A Review of the Decomposition Method in Applied Mathematics. Academic Press, Inc, 135: 501-544.

[2] Arpaci, S. Vedat 1966. Conduction heat transfer. Addison Wesley Publishing Company, Massachusetts.

[3] Benabidallah, M. and Cherruault, Y. (2004). Application of the Adomian method for solving a class of boundary problems. Kybernetes, 33 (1): 118-132.

[4] Cui, M. and Geng, F. 2007. Solving singular two-point boundary value problem in reproducing kernel space. Journal of Computations and Applied Mathematics, 205: 6-15.

[5] Duan, J.-S, Rach, R., Baleanu, D. and Wazwaz, A.-M. 2012. A review of the Adomian decomposition method and its applications to fractional differential equations. Commun. Frac. Calc., 3 (2): 73-99.

[6] Ebaid, A. 2010. Modification of Lesnic's Approach and New Analytic Solutions for Some Nonlinear Second-Order Boundary Value Problems with Dirichlet Boundary Conditions. Zeitschrift $f^{\prime \prime}$ ur Naturforschung, Tubingen, 65a: 692-296.

[7] Hasan, Y. Q. and Zhu, L. M. 2008. Modified Adomian decomposition method for singular initial value problems in the second order ordinary differential equations. 3 (1): 183 193.

[8] Hasan, Y. Q. and Zhu, L. M. 2009. A note on the use of modified Adomian decomposition method for solving singular boundary value problems of higher-order ordinary differential equations. Communications in Nonlinear Science and Numerical Simulation, 14 (8): 3261-3265.

[9] Inc, M. and Evans, D. J. 2003. The decomposition method for solving a class of singular two- point boundary value problems. International Journal of Computational Mathematics. 80 (7): 869-882.

[10] Kaliyappan, M. and Hariharan, S. 2015. Symbolic computation of Adomian polynomials based on Rach's Rule. British Journal of Mathematics and Computer Science, 5 (5): 562-570.

[11] Khuri, S. A. and Sayfy, A. 2010. A novel approach for the solution of a class of singular boundary value problems arising in physiology. Mathematical and Computer Modeling, 52 (3): 626-636.

[12] Kim, W. and Chun, C. 2010. A modified Adomian decomposition method for solving higher- order singular boundary value problems. Z. Naturforsch, 65a: 1093-1100.

[13] Kraus, A. D., Aziz, A. and Welty, J. 2001. Extended Surface Heat Transfer, John Wiley and Sons, Inc., New York.

[14] Kumar, M. 2002. A three-point finite difference method for a class of singular two-point boundary value problems. Journal of Computations and Applied Science, 145: 89-97.

[15] Lesnic, D. 2001. A computational algebraic investigation of the decomposition method for time-dependent problems. Elsevier Science Inc., 119: 197-206. 
[16] Lin, Y. and Chen, C. K. 2014. Modified Adomian decomposition method for double singular boundary value problems. Rom. Journal of Physics, Bucharest, 59 (5-6): 443454.

[17] Mokheimer Esmail, M. A. 2003. Heat transfer from extended surfaces subject to variable heat transfer coefficient. SpringerVerlag, 39: 131-138.

[18] Noor, M. A. and Mohyud-Din, S. T. 2008. Solution of singular and non-singular initial and boundary value problems by modified variational iteration method. Hindawi Publishing Corporation, 1-23.

[19] Ravi, A. S. V. and Aruna, K. 2008. Solution of singular two- point boundary value problems using differential transformation method. (A372): 4062-4066.

[20] Singh, R., Kumar, J., and Nelakanti, G. 2012. New approach for solving a class of doubly singular two-point boundary value problems using Adomian decomposition method. Hindawi Publishing Corporation, 1-23.

[21] Wazwaz, A.-M. 1999. A Reliable Modification of Adomian Decomposition Method. Applied Mathematics Computation, 102 (1): 77-86.

[22] Zavalani, G. 2015. Galerkin-finite element method for two point boundary value problems of ordinary differential equations. Science Publishing Group, 4 (2): 64-68. 